JOURNAL OF

APPLIED

CRYSTALLOGRAPHY

ISSN 1600-5767

Received 15 September 2015

Accepted 8 January 2016

Edited by V. T. Forsyth, Institut Laue-Langevin, France, and Keele University, UK

Keywords: neutron transmission; mosaic crystals; full-pattern analysis.

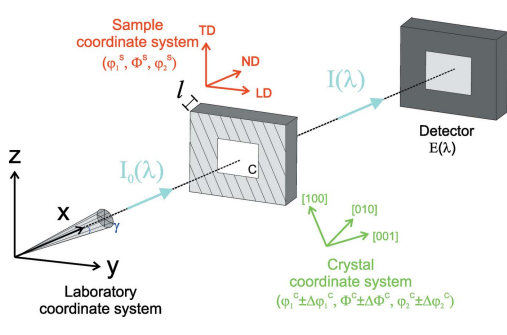

C 2016 International Union of Crystallography

\section{Full-pattern analysis of time-of-flight neutron transmission of mosaic crystals}

\author{
Florencia Malamud* and Javier R. Santisteban
}

Laboratorio de Física de Neutrones, Centro Atómico Bariloche, Avenida Bustillo 9500, San Carlos de Bariloche, Rio
Negro 8400, Argentina. *Correspondence e-mail: fmalamud@cab.cnea.gov.ar

The energy-resolved neutron transmission of mosaic crystals contains a series of dips in intensity, at specific neutron wavelengths defined by the orientation of the specimen in the neutron beam. This article presents a Rietveld type fullpattern analysis of neutron transmission experiments on mosaic crystals performed at spallation pulsed neutron sources. The proposed analysis provides precise and simple determination of lattice parameters, mosaicity, extinction factors and crystal orientation, and is especially suited to investigate the spatial variation of such microstructural information across macroscopic specimens with $\sim 1 \mathrm{~mm}$ resolution. The effect of extinction on the intensity of Bragg reflections has been successfully accounted for by a parameter measuring the ratio of the beam attenuation due to Bragg reflection to the combined attenuation due to absorption and scattering processes. Experiments were performed at the ENGIN-X beamline, ISIS Facility, UK, on several naturally occurring and man-made mosaic crystals, including a copper monochromator at temperatures between 55 and $300 \mathrm{~K}$, an iron-nickel meteorite, and a natural pyrite crystal. Typical experimental resolutions found for lattice parameters and mosaicity are 0.03 and $7 \%$, respectively. The possibilities of the technique for quantitative phase and/or texture analysis of specimens composed of several grains or phases are discussed.

\section{Introduction}

Energy-resolved neutron imaging is a growing field that exploits the dependence of the attenuation of neutron beams on the energy of the incoming neutrons. In particular, for thermal and cold neutrons passing through crystalline materials, the attenuation is strongly dependent on wavelength owing to Bragg diffraction on the crystal planes. Moreover, as elastic diffraction of the neutrons can occur over several length scales going from $10^{-11}$ to $10^{-7} \mathrm{~m}$, neutron radiographies of polycrystalline objects can reveal many details about the underlying microstructure of the material. Images taken with different neutron energies can be combined to give contrast factors sensitive to specific crystallographic phases (Steuwer et al., 2004), texture components (Santisteban et al., 2012), or sizes of pores or grains (Strobl et al., 2008). In instruments having high wavelength resolution $(\delta \lambda / \lambda \simeq 0.001)$, it is even possible to produce quantitative images of properties such as lattice parameters (Santisteban et al., 2002), phase volume fractions and elastic strain (Tremsin et al., 2012). High resolution on the neutron wavelength scale is mostly achieved by using time-of-flight (TOF) techniques in pulsed neutron sources (Santisteban et al., 2006; Harjo et al., 2010; Kockelmann et al., 2013), or by the use of double monochromator arrangements (Treimer et al., 2006) or choppers (Strobl et al., 2012) in nuclear reactors. 
Energy-resolved neutron imaging has been traditionally applied to polycrystalline materials, where the crystalline structure is revealed through Bragg edges. These are sharp discontinuities that appear in the wavelength-dependent neutron transmission as a result of coherent elastic scattering on the lattice planes These Bragg edges occur because, for a given $h k l$ reflection, the Bragg angle increases as the wavelength increases until $2 \theta$ is equal to $180^{\circ}$. At wavelengths greater than this critical value no scattering by this particular $\{h k l\}$ family can occur, and there is thus an increase in transmitted intensity at $\lambda=2 d_{h k l}$. Hence, precise definition of the position of Bragg edges provides a measure of lattice parameters, whilst the height of these edges gives information about phase volume fractions (Woracek et al., 2014; Makowska et al., 2015) and texture effects (Kockelmann et al., 2007; Santisteban et al., 2012; Malamud et al., 2014). The height and position of the edges is usually defined by performing least-squares fits to the measured transmitted spectrum $T(\lambda)$. For better accuracy in the refined lattice parameter, several Bragg edges are simultaneously measured in TOF instruments, and the information about lattice parameters and crystallographic phases is obtained by performing full-pattern Rietveld type analysis of the transmitted spectrum (Vogel, 2000; Kiyanagi et al., 2012). When only lattice parameter information is required, a full-pattern Pawley type analysis that constrains edge positions but leaves edge intensities unconstrained becomes more efficient (Steuwer et al., 2003), as it can be applied to textured materials without the need to specifically model the orientation distribution function of the crystallites composing the material.

In principle, it is possible to apply wavelength-resolved neutron imaging methods to single-crystal objects such as meteorites or turbine blades. Yet quantitative analysis and interpretation of contrast factors are not straightforward because crystallographic effects are more complex in this case. The wavelength-dispersive neutron transmission of single crystals contains a series of dips in intensity, appearing at specific neutron wavelengths, due to reflection on individual crystallographic planes. The position, depth and width of those dips on the wavelength scale depend on the neutronic properties of the material, the degree of perfection of the single crystal and the orientation of the specimen in the neutron beam. In previous work (Santisteban, 2005), we studied the positions and widths of those dips for a $\mathrm{Cu}$ mosaic crystal used as a neutron monochromator, in order to measure the spatial variation of lattice parameters, orientation and mosaicity across the crystal, yet with limited spatial resolution. The intensity of the dips was not analysed in that work because it was not required to extract the lattice parameters, orientation and mosaicity, and it is in fact a complex function of the variables mentioned above. The analysis performed in that work was based on post-processing of the positions and widths of the dips, previously obtained from least-squares fits to isolated dips observed in the neutron spectrum. Such an approach limited the number of reflections that could be effectively incorporated in the analysis, as severe overlapping occurs at short neutron wavelengths owing to the large number of crystal families having similar $d$ spacing. Moreover, mapping of the spatial variation of mosaicity and orientation across a specimen using the analysis presented in that work was complicated owing to the several steps required to process the data, which changed for each position and orientation of the specimen investigated.

Here we present a method for the full-pattern analysis of the wavelength-dependent transmission of imperfect single crystals. The analysis includes an extinction factor that describes the intensity of neutrons scattered by the individual crystallographic planes. The method has been applied to study three different single-crystal objects measured at the ENGIN$\mathrm{X}$ beamline, ISIS Facility, UK. These objects include (i) a large copper monochromator, at temperatures between $55 \mathrm{~K}$ and room temperature, (ii) an iron-nickel meteorite from the Sikhote-Alin fall, and (iii) a relatively large natural pyrite crystal. The method will be useful to achieve quantitative energy-resolved neutron imaging of single-crystal objects.

The paper is organized as follows. In $\S 2$ we introduce the theoretical basis of neutron transmission experiments in imperfect mosaic crystals. In $\$ 3$ we briefly describe the implementation of the proposed technique within the MATLAB (The MathWorks Inc., Natick, MA, USA) computing system. In $\S 4$ we describe the experiments and the analyses performed on the specimens. In $\$ 5$ we discuss the capabilities and limitations of the technique. Finally, in $\S 6$ we summarize the main scientific contributions of the present work.

\section{Theory}

Fig. 1 shows a schematic representation of a neutron transmission experiment on a mosaic crystal. A collimated polychromatic neutron beam, with a lateral cross section of $C$, angular divergence $\gamma$ and differential flux $I_{0}(\lambda)$ (in neutrons per $\mathrm{cm}^{2}$ per second per steradian per ångström), impacts on a mosaic crystal specimen and emerges on the other side with a

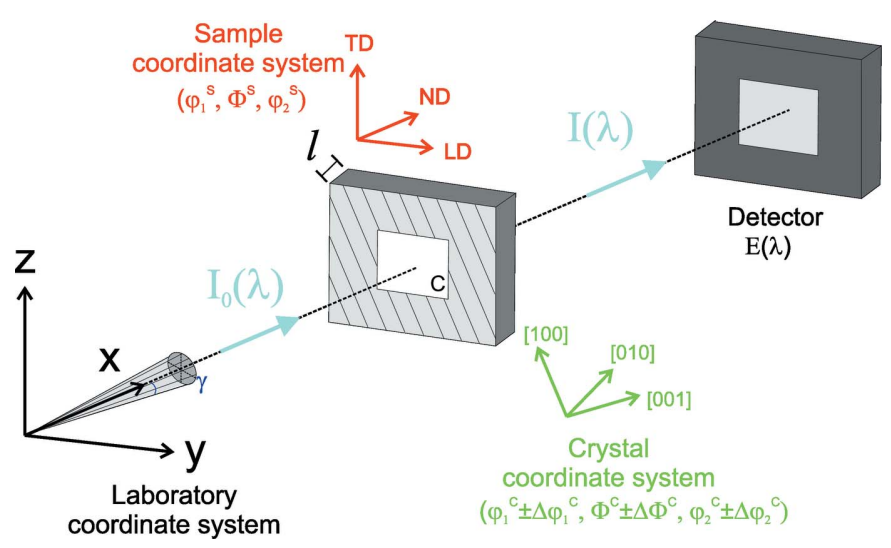

Figure 1

Schematic representation of a neutron transmission experiment on a mosaic crystal, where $C$ and $\gamma$ represent the neutron beam cross section and angular divergence, respectively. Coordinate systems attached to the laboratory, the sample and the crystal are plotted in different colours. 
differential flux $I(\lambda)$, which is measured by a spectroscopic detector of efficiency $E(\lambda)$. The cross sectional area of the specimen is larger than the incident beam. The arrangement is equivalent to a single-crystal Laue experiment, but the neutrons reflected out of the beam are counted through the 'fingerprint' they leave on the transmitted neutron spectrum rather than by the spots recorded on a film in diffraction geometry.

By a mosaic crystal specimen we mean a single crystal whose crystalline orientation is represented by the three Euler angles $\left(\varphi_{1}^{\mathrm{C}}, \Phi^{\mathrm{C}}, \varphi_{2}^{\mathrm{C}}\right)$ in the laboratory system, yet having a certain substructure that produces small deviations around those Euler angles; these are described by Gaussian distributions of widths $\left(\Delta \varphi_{1}^{\mathrm{C}}, \Delta \Phi^{\mathrm{C}}, \Delta \varphi_{2}^{\mathrm{C}}\right)$, respectively. Macroscopically, the mosaic crystal is a parallelepiped of dimensions $\left(l_{\mathrm{ND}}, l_{\mathrm{TD}}, l_{\mathrm{LD}}\right)$ along the normal, transverse and longitudinal directions, respectively. The macroscopic orientation of the specimen in the laboratory system is given by the three Euler angles $\left(\varphi_{1}^{\mathrm{S}}, \Phi^{\mathrm{S}}, \varphi_{2}^{\mathrm{S}}\right)$. The thickness of the specimen along the neutron beam direction is $\mathbf{I}$, which for the specimen orientation shown in the figure equals $l_{\mathrm{ND}}$. A rotation of the specimen changes the thickness $\mathbf{I}$, the Euler angles, and the overall intensity and shape of the transmitted neutron beam $I(\lambda)$.

The experimental procedure is very simple. It consists of measuring the spectroscopic transmission of the specimen, $T(\lambda)$, by comparing the signal recorded by the detector over a certain counting time $T_{\mathrm{c}}$ when the crystal is in the beam, $i(\lambda)=$ $I(\lambda) C \gamma T_{\mathrm{c}} E(\lambda) \Delta \lambda$ (assuming no background counts), with the direct signal recorded after removing the specimen from the neutron beam, $i_{0}(\lambda)=I_{0}(\lambda) C \gamma T_{\mathrm{c}} E(\lambda) \Delta \lambda$ :

$$
T(\lambda)=i(\lambda) / i_{0}(\lambda) .
$$

The fraction of neutrons removed from the incident beam by the specimen is given by $[1-T(\lambda)]$. Fig. $2(a)$, taken from Santisteban (2005), shows this magnitude for a copper monochromator. As identified in the figure, neutrons removed from the beam are either absorbed by the nuclei $(A)$, scattered $(S)$ or Bragg-reflected on the crystal planes $(R)$ :

$$
1-T=A+S+R .
$$

The contributions due to neutron absorption and scattering have a smooth dependence on neutron wavelength. By contrast, the Bragg-reflected contribution $R$, shown in Fig. 2(b), is composed of a large number of sharp peaks, one for every neutron wavelength $\lambda_{h k l}$ that fulfils the Bragg condition for a certain $(h k l)$ plane of the single crystal. The peaks in $R(\lambda)$ compose a diffraction pattern that contains a wealth of information about the microscopic properties of the specimen, easily accessible by energy-resolved neutron imaging experiments. Such information can be extracted by performing a quantitative Rietveld type least-squares analysis of the $R(\lambda)$ diffractogram, based on a sound physical model for the transmitted signal. For this purpose, the $R(\lambda)$ diffractogram can be simply described by a sum series of peaks located at wavelengths $\lambda_{h k l}$, each one having an integrated area $I_{h k l}$ and an FWHM $\varpi_{h k l}$ :

$$
R(\lambda)=\sum_{h k l} I_{h k l} P\left(\lambda_{h k l}, \varpi_{h k l}, \lambda\right),
$$

where the function $P\left(\lambda_{h k l}, \varpi_{h k l}, \lambda\right)$ describes the actual peak shape (which depends on the instrument) and has unit area, i.e.

$$
\int_{0}^{\infty} P\left(\lambda_{h k l}, \varpi_{h k l}, \lambda\right) \mathrm{d} \lambda=1 .
$$

Within the kinematical theory of diffraction it is assumed that the probability of a neutron being removed from the beam is proportional to an attenuation coefficient $\mu$, and the removed beam is simply

$$
1-T=1-\exp (-\mu l) \text {. }
$$

As discussed above, different processes contribute to the attenuation coefficient:

$$
\mu(\lambda)=\mu_{\mathrm{A}}(\lambda)+\mu_{\mathrm{S}}(\lambda)+\mu_{\mathrm{R}}(\lambda),
$$

where the subscripts $\mathrm{A}, \mathrm{S}$ and $\mathrm{R}$ refer to absorption, scattering and Bragg-reflection processes, respectively. The absorption contribution to the attenuation coefficient is given by

$$
\mu_{\mathrm{A}}(\lambda)=N \sigma_{\mathrm{abs}}\left(\lambda / \lambda_{0}\right),
$$

with $N$ the number of atoms per unit volume and $\sigma_{\text {abs }}$ the microscopic absorption cross section of the atom at the wavelength $\lambda_{0}$ (typically $\lambda_{0}$ corresponds to a neutron energy of $25 \mathrm{meV}$, e.g. $\left.\lambda_{0}=1.8 \AA\right)$. The scattering contribution $\mu_{\mathrm{S}}(\lambda)$ to

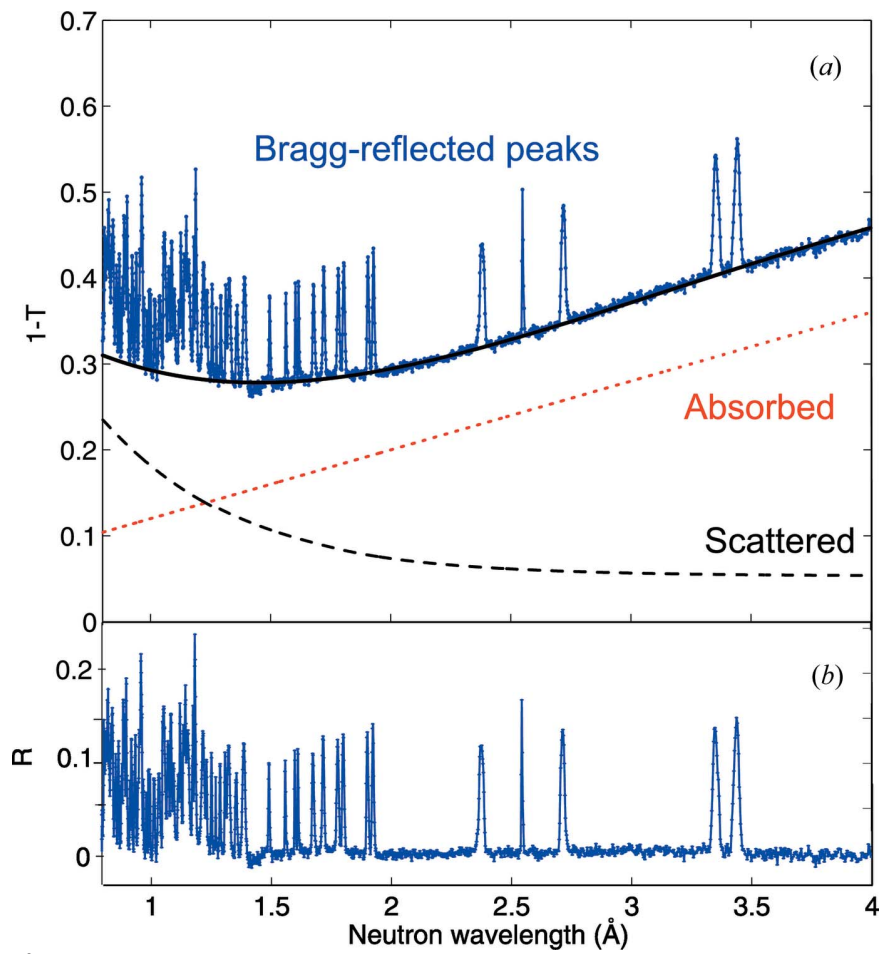

Figure 2

Contributions to the attenuation of a neutron beam in a mosaic crystal. (a) The blue solid-dotted line represents the experimental data for a $\mathrm{Cu}$ crystal along the [110] direction. The red dotted and black dashed lines are the nuclear absorption and thermal diffuse scattering, respectively, and the solid black line is the sum of both contributions. (b) The Braggreflected contribution $R$ composed of a large number of sharp peaks. 
the attenuation coefficient includes a combination of incoherent scattering processes and inelastic scattering. This contribution is large for hydrogenous materials and increases with the specimen temperature $T$. Compact theoretical expressions to calculate this contribution have been given by Granada (1984):

$$
\mu_{\mathrm{S}}(\lambda)=N \sigma_{\mathrm{S}}\left(\lambda, \sigma_{\mathrm{coh}}, \sigma_{\mathrm{inc}}, \mathcal{A}, \Theta_{\mathrm{D}}, T\right),
$$

where $\mathcal{A}, \sigma_{\text {coh }}$ and $\sigma_{\text {inc }}$ are, respectively, the atomic mass and the coherent and the incoherent cross section of the scattering nucleus. The Debye temperature $\Theta_{\mathrm{D}}$ incorporates the effect of the thermal vibrations of the nuclei in the scattering function. The expression for $\mu_{\mathrm{S}}(\lambda)$ is presented in Appendix $A$.

In order to evaluate $\mu_{\mathrm{R}}(\lambda)$, the Bragg-reflected contribution to the attenuation coefficient, we note that it can be written as the sum of the $\mu_{\mathrm{R}}^{h k l}$ contributions due to beam reflection on each crystallographic plane. The attenuation coefficient associated with each reflection is obtained by recalling the expression for the integrated reflectivity of the $h k l$ reflection for the Laue experiment within the kinematical theory of diffraction (Zachariasen, 1945):

$$
\mu_{\mathrm{R}}^{h k l}=\frac{\left|F_{h k l}\right|^{2} \lambda_{h k l}^{4}}{2 V^{2} \sin ^{2} \theta_{h k l}}
$$

where $\left|F_{h k l}\right|$ is the structure factor (including the DebyeWaller factor), $V$ is the volume of the unit cell and $\theta_{h k l}=\sin ^{-1}\left(\lambda_{h k l} / 2 d_{h k l}\right)$ is the Bragg angle for the reflection. Within this approximation, the integrated reflectivity of the $h k l$ peak is given by $I_{h k l}=1-\exp \left(-l \mu_{\mathrm{R}}^{h k l}\right)$, which for a thin specimen $\left(l \ll 1 / \mu_{\mathrm{R}}^{h k l}\right)$ becomes $I_{h k l} \simeq l \mu_{\mathrm{R}}^{h k l}$. Hence, a first approximation to the Bragg-reflected attenuation $R(\lambda)$, i.e. the fraction of incident neutrons that are reflected on Bragg planes, as displayed in Fig. 2(b), is

$$
R_{\text {kinem }}(\lambda)=l \sum_{h k l} \mu_{\mathrm{R}}^{h k l} P\left(\lambda_{h k l}, \varpi_{h k l}, \lambda\right) .
$$

In practice the integrated intensity of the Laue spot is smaller than the area predicted by the kinematical theory owing to the extinction of the neutron beam (Bacon \& Lowde, 1948; Zachariasen, 1969). This is because as the incident neutron beam travels through a large crystal its amplitude is reduced by absorption, scattering and reflection, so the inner parts of the crystal contribute less to the diffracted intensity than the more external regions. Moreover, in a single crystal the attenuation of the forward travelling wave due to Bragg reflection may be regarded as being a result of repeatedly reflected components thrown into the same direction with opposite phase. So, in large crystals the interaction between the incident and scattered beams must be taken into account, and equation (9) is no longer valid. The extinction of the neutron (or X-ray) beam within a large crystal is the subject of the dynamical theory of diffraction, and its theoretical solution for very different experimental conditions (type of radiation, specimen geometry, crystal perfection etc) has been treated by many authors since the formulation of the problem by Darwin in 1922 (Darwin, 1922; Hamilton, 1957; Zachariasen, 1967; Bacon \& Lowde, 1948; Sears, 1997; etc). Here we consider the effects of extinction empirically, by simply introducing an extinction factor $y_{h k l}$, representing the ratio between the integrated intensity predicted by the kinematical theory and the actual value measured by the experiment. So equation (10) becomes

$$
R(\lambda)=l \sum_{h k l} y_{h k l} \mu_{\mathrm{R}}^{h k l} P\left(\lambda_{h k l}, \varpi_{h k l}, \lambda\right) .
$$

As we will show later, under some circumstances the extinction factor so defined has a relatively simple dependence on the ratio between the attenuation coefficient due to Bragg reflection and the coefficients due to absorption and scattering.

\section{Least-squares analysis of TOF transmission experiments}

TOF neutron transmission experiments are usually performed at pulsed neutron sources. Short pulses of high-energy neutrons are brought to thermal energies through collisions within a moderator material. A collimated neutron beam is extracted from the moderator to be used in transmission or scattering experiments. The wavelength of the neutrons emerging from the moderator is easily determined by recording the time of flight $t$ taken by the neutron to travel the distance $L$ between the moderator and the detector, i.e.

$$
\lambda=\frac{h}{m L}\left(t-t_{0}\right),
$$

with $h$ Planck's constant, $m$ the neutron mass and $t_{0}$ a delay time, representing the average time spent by the neutrons within the moderator and possible delays of the detection system. The $(1-T)$ spectrum shown in Fig. 2(a) was actually measured on time-of-flight scale and subsequently transformed into wavelength scale using equation (12). The experimental Bragg-reflected attenuation $R(\lambda)$ can be empirically extracted from the measured $(1-T)$ simply by subtraction of a smooth background from the measured spectrum. For the least-squares analysis, we have adopted an approximation to the $A(\lambda)+S(\lambda)$ contribution using a polynomial baseline fit on experimental data, as will be explained later on.

The experimental Bragg-reflected attenuation $R(\lambda)$ pattern consists of a collection of individual peak profiles, each of which has a position $\left(\lambda_{h k l}\right)$, a width $\left(\varpi_{h k l}\right)$ and an integrated area $\left(I_{h k l}\right)$. We propose to perform a full-pattern least-squares analysis of the experimental $R(\lambda)$ by implementing a physically based analytical expression of equation (11). This is essentially the method proposed by Rietveld (1969), where a least-squares refinement is carried out until the best fit is obtained between the entire observed pattern and the entire calculated pattern based on the simultaneously refined model parameters. This approach is very efficient as it allows extraction of useful information from overlapping peaks. Moreover, the correlations that exist between the parameters describing different peaks translate into a lower number of fitting parameters and hence a more robust least-squares 
procedure. Below, we provide analytical expressions describing the dependence of peak shape $P$, peak position $\lambda_{h k l}$ and peak broadening $\varpi_{h k l}$ on the microstructural properties of the specimen and on the details of the experimental arrangement presented in Fig. 1.

\subsection{Peak profile function}

The resolution function in TOF experiments performed at accelerator-based neutron sources is typically asymmetric, as a result of the convolution of the distribution of time spent by the neutrons inside the moderator $\left[t_{0}\right.$ in equation (12)] and the geometric uncertainties in the actual distance travelled by the detected neutron [ $L$ in equation (12)]. In this work we adopt one of the simplest models for the resolution function (Kropff et al., 1982), which represents the geometrical deviations by a Gaussian of deviation $v_{h k l}(\lambda)$ and uses a truncated decaying exponential of constant $\tau_{h k l}(\lambda)$ to describe the uncertainty of the emission time of the neutron. The expression for a peak located at wavelength $\lambda_{h k l}$ is

$$
\begin{aligned}
P\left(\lambda_{h k l},\left[\tau_{h k l}, v_{h k l}\right], \lambda\right)= & \frac{1}{\tau_{h k l}(\lambda)} \exp \left[-\frac{\lambda-\lambda_{h k l}}{\tau_{h k l}(\lambda)}\right] \\
& \otimes \frac{1}{(2 \pi)^{1 / 2} v_{h k l}(\lambda)} \exp \left[-\frac{\left(\lambda-\lambda_{h k l}\right)^{2}}{2 v_{h k l}(\lambda)^{2}}\right] \\
= & \frac{1}{2 \tau_{h k l}(\lambda)} \exp \left[-\frac{\lambda-\lambda_{h k l}}{\tau_{h k l}(\lambda)}+\frac{v_{h k l}(\lambda)^{2}}{2 \tau_{h k l}(\lambda)^{2}}\right] \\
& \times \operatorname{erfc}\left[-\frac{\lambda-\lambda_{h k l}}{2^{1 / 2} v_{h k l}(\lambda)}+\frac{v_{h k l}(\lambda)}{\tau_{h k l}(\lambda)}\right] .
\end{aligned}
$$

This peak profile allows a simple deconvolution of the instrument and sample contributions to the resolution, simplifying the quantification and interpretation of the observed peak broadening. On the other hand, the FWHM of this peak shape does not have a simple analytical expression as a function of $v_{h k l}, \tau_{h k l}$ and $\lambda_{h k l}$.

\subsection{Peak positions}

The sharp peaks in the Bragg-reflected component $R(\lambda)$ appear at precise wavelengths specified by Bragg's law,

$$
\lambda_{h k l}=2 d_{h k l} \sin \theta_{h k l},
$$

where $d_{h k l}$ is the interplanar distance for the $(h k l)$ planes and $\theta_{h k l}$ is the Bragg angle. Provided the Bragg angles depend on the relative orientation between the neutron beam and the crystal, so do the wavelengths at which the peaks in $R(\lambda)$ are observed. Therefore, the positions of the diffraction peaks depend on the orientation between the incident beam and the crystal. In particular, for a cubic crystal with lattice parameter $a$, the $h k l$ reflection diffracts neutrons of wavelength

$$
\lambda_{h k l}=2 a \frac{\left|h a_{11}+k a_{12}+l a_{13}\right|}{h^{2}+k^{2}+l^{2}},
$$

where $a_{11}, a_{12}$ and $a_{13}$ are the direction cosines of the incident neutron beam in the coordinate system of the crystal. Strictly speaking, only two direction cosines are independent, as they are related by the normalization condition $a_{11}^{2}+a_{12}^{2}+a_{13}^{2}=1$.
An alternative expression of Bragg's law, useful for the interpretation of the transmitted spectrum, is

$$
\lambda_{h k l}=2 d_{h k l} \cos \alpha_{h k l},
$$

where $\alpha_{h k l}$ is the angle between the neutron beam and the normal to the reflecting crystal planes. Hence, the peaks observed in $R(\lambda)$ correspond to the projections of all interplanar distances $d_{h k l}$ along the direction of the incident neutron beam.

\subsection{Peak widths}

The main contribution to the width $v_{h k l}(\lambda)$ of the Gaussian component of the instrument resolution comes from the uncertainty in the neutron wavelength $\Delta \lambda_{h k l}$ of the reflected neutrons. For an ideal perfect crystal only neutrons of a single wavelength $\lambda_{h k l}$ are removed from the beam, and the width of the peak would correspond to the moderator distribution $\tau_{h k l}(\lambda)$. However, for a mosaic crystal the Bragg condition is relaxed, as a finite distribution of crystal orientation and interplanar distances exists within the crystal. Hence, from Bragg's law

$$
\left(\frac{\Delta \lambda_{h k l}}{\lambda_{h k l}}\right)^{2}=\left(\frac{\Delta d_{h k l}}{d_{h k l}}\right)^{2}+\left(\Delta \alpha_{h k l}\right)^{2} \tan ^{2} \alpha_{h k l},
$$

where we have assumed that there is no correlation between $d_{h k l}$ and $\alpha_{h k l}$. The first term $\overline{\varepsilon^{2}}=\left(\Delta d_{h k l} / d_{h k l}\right)^{2}$ represents the mean-square elastic deformation of the crystal lattice, whilst the second term $\left(\Delta \alpha_{h k l}\right)^{2}$ reflects the finite distribution of lattice orientations in the light of a finite beam divergence. The angular uncertainty arises from the misalignment of mosaic blocks as well as from the divergence of the incident beam. In the general case, both the incident beam divergence and mosaic block misalignment are anisotropic, so the angular uncertainty $\Delta \alpha_{h k l}$ depends on the actual orientation of the diffraction plane for each $h k l$ reflection. In the present analysis, as a first approximation to the problem, we have adopted isotropic misorientation distributions for both the incident beam divergence and the crystal mosaic blocks $\left(\Delta \varphi_{1}^{\mathrm{C}}=\Delta \Phi^{\mathrm{C}}=\Delta \varphi_{2}^{\mathrm{C}}\right)$, represented by Gaussian distributions of widths $\gamma$ and $\eta$, respectively. The mosaicity $\eta$ gives a measure of the misorientation of the composing crystal blocks, and for mosaic crystals it is much larger than the intrinsic Darwin (1922) width. In monochromator studies, the mosaicity is typically measured by rocking curves at constantwavelength diffractometers. Within this approximation, the angular uncertainty does not depend on the $h k l$ reflection:

$$
\left(\Delta \alpha_{h k l}\right)^{2}=(\Delta \alpha)^{2}=\gamma^{2}+\eta^{2} .
$$

So, in the present model the Gaussian contribution to the Bragg reflected peaks for any $h k l$ reflection can be accounted for by a single parameter $v_{h k l}$ given by

$$
v_{h k l}(\lambda)^{2}=\lambda_{h k l}^{2}\left[\varepsilon^{2}+\left(\gamma^{2}+\eta^{2}\right) \tan ^{2} \alpha_{h k l}\right]
$$

On the other hand, the asymmetric contribution to the peak width does not involve crystallographic effects, but depends on the specific $h k l$ reflection through the neutron wavelength: 


$$
\tau_{h k l}=\tau\left(\lambda=\lambda_{h k l}\right)
$$

The function $\tau(\lambda)$ is a characteristic of the moderator, and it has a smooth dependence on neutron wavelength, reaching a plateau at long wavelengths (Kropff et al., 1982).

\subsection{MATLAB implementation}

We have produced a library within the MATLAB programming environment, designed to analyse neutron transmission experiments in mosaic crystals. This code is freely available and can be requested from the authors. The library incorporates the equations presented in the previous sections, but so far only for cubic crystals. From equations (11), (13), (15) and (19) we see that $R(\lambda)$ can be described by a multipeak fitting algorithm which is applied to the experimental data using a limited number of fitting parameters, namely, the lattice parameter $a$, the direction cosines $a_{11}$ and $a_{12}$, the meansquare strain $\varepsilon$, the mosaicity $\eta$, and the extinction factors $y_{h k l}$. On the other hand, the smooth dependence on wavelength of the $A(\lambda)+S(\lambda)$ contribution to the $[1-T(\lambda)]$ signal can be effectively described as a polynomial baseline. The library developed in this work can perform the following tasks:

3.4.1. Kinematical transmission calculation. The library calculates the wavelength-dependent transmission $T(\lambda)$ for a mosaic crystal with a certain orientation in the laboratory system, according to the kinematic theory, as given by equations (5)-(9), (13), (15), (19) and (20).

3.4.2. Bragg scattering component extraction. It extracts the Bragg scattering component $R(\lambda)$ from the experimental $[1-T(\lambda)]$ spectrum (Fig. $2 b$ ). This is achieved by subtracting an approximation to the $A(\lambda)+S(\lambda)$ contribution, represented by a five-degree polynomial baseline fit on experimental data.

3.4.3. Indexing. The second step in the analysis is peak indexing. The code uses the method described in Appendix $A$ of Santisteban (2005), which exploits the existence of a cutoff wavelength at $\lambda_{h k l}^{\max }=2 d_{h k l}$ for each crystal family. The method produces an ordered list $\left(2 d_{\mathrm{I}}>2 d_{\mathrm{II}}>2 d_{\mathrm{III}}>\right)$ determining the ranges where different plane families can operate.

3.4.4. Least-squares full-pattern analysis. After indexing, the full-pattern least-squares refinement is applied to the whole Bragg-reflected component profile to refine the lattice parameter $(a)$, the crystal orientation $\left(a_{11}, a_{12}\right)$, the meansquare elastic deformation of the crystal lattice $\left(\varepsilon^{2}\right)$, the crystal mosaicity $(\eta)$ and the extinction factors $\left(y_{h k l}\right)$. The fitting parameters are obtained by least-squares fitting to the experimental data, using a multi-peak fitting algorithm based on equation (11). Typical fits are shown in Figs. 4, 7 and 8 for a copper monochromator, an iron-nickel meteorite and a pyrite crystal, respectively, as described in the next section. The fitting routine uses the MATLAB Optimization Toolbox (http://www.mathworks.com/products/optimization/) for an efficient least-squares minimization process.

3.4.5. Laboratory-to-crystal-system rotation matrix calculation. The transformation matrix between the laboratory and crystal systems can be obtained by measuring the spectra transmitted by a specimen at two different orientations, differing just by a small rotation around the vertical direction of the laboratory system (the $z$ axis in Fig. 1) (Santisteban, 2005). Let $\mathbf{v}_{1}$ and $\mathbf{v}_{2}$ be the coordinates of the neutron beam in the crystal system measured for each orientation, as given by the direction cosines refined in each case. Provided $\mathbf{v}_{1}$ and $\mathbf{v}_{2}$ are on the $X Y$ plane of the laboratory system, and by setting the laboratory $X$ axis to be along $\mathbf{v}_{1}$, the direction cosines of the laboratory $Y$ and $Z$ axes in the coordinate system of the crystal are given by

$$
\begin{aligned}
& \hat{\mathbf{x}}=\mathbf{v}_{1}=\left(a_{11}, a_{12}, a_{13}\right), \\
& \hat{\mathbf{z}}=\mathbf{v}_{1} \times \mathbf{v}_{2}=\left(a_{31}, a_{32}, a_{33}\right), \\
& \hat{\mathbf{y}}=\hat{\mathbf{z}} \times \mathbf{v}_{1}=\left(a_{21}, a_{22}, a_{23}\right),
\end{aligned}
$$

which implicitly defines the transformation matrix $a_{i j}$ between the crystal and laboratory coordinate system (Bunge, 1982). On the other hand, the transformation matrix between the laboratory system and the macroscopic coordinate system of the specimen (normal, transverse and longitudinal directions: ND, TD and LD) is defined by optical means during the alignment of the specimen in the neutron beam. Using these equations, the package can perform any transformation between the three coordinate systems displayed in Fig. 1 (Hielscher \& Schaeben, 2008).

\section{Experiments}

We have performed a series of energy-resolved neutron transmission experiments on three man-made and naturally occurring mosaic crystals, in order to determine their lattice parameters, crystal orientation, mosaicity and extinction factors using the method presented in the previous section. The specimens investigated included a copper crystal used as a neutron monochromator, an iron-nickel meteorite from the Sikhote-Alin fall (Russia) and a $15 \times 15 \times 15 \mathrm{~mm}$ pyrite crystal. The experiments were performed with the ENGIN-X instrument at the ISIS Facility, Rutherford Appleton Laboratory, UK. For all specimens, transmission spectra were taken at four slightly different orientations in order to (i) assess the accuracy of the refined parameters and (ii) define the precise crystal orientation. Two orientations are required to define the precise values of the Euler angles, but this number was doubled in order to assess the stability of the refined parameters against different experimental conditions.

In all cases the goodness of the refinements has been quantified by means of a parameter $G$, defined as

$$
G=100 \frac{\sum_{i}\left|y_{i}(\mathrm{obs})-y_{i}(\mathrm{cal})\right|}{\left(N_{\mathrm{obs}}-N_{\mathrm{p}}\right)},
$$

where $y_{i}$ (obs) is the observed intensity at the $i$ th wavelength interval, $y_{i}(\mathrm{cal})$ the calculated intensity, $N_{\text {obs }}$ the number of observations and $N_{\mathrm{p}}$ the number of fitting parameters. This $G$ parameter estimates the agreement between the observed and calculated $R(\lambda)$ profiles. Provided the adjusted $y$ values are normalized between 0 and 1 , the $G$ parameter is expressed in $\%$ of the incident neutron beam. So it can be directly 
compared with the typical statistical uncertainty of the measurement.

\subsection{ENGIN-X description}

The ENGIN-X instrument is a TOF neutron diffractometer optimized for strain measurements, but also used for neutron transmission experiments. Detailed information about the instrument is given by Santisteban et al. (2006), so only a brief description is provided here. ENGIN-X uses a polychromatic neutron beam produced by a liquid methane moderator, which is transported to the sample position along a curved $50 \mathrm{~m}$-long neutron guide. The polychromatic neutron spectrum incident on the specimen is shown in Fig. 3. For the present experiments, the statistical uncertainty of the measured transmission is dependent on the wavelength interval. Typical values were $4.5 \%$ in the range $[0.5,1.5] \AA$, $3.5 \%$ in the range $[1.5,4] \AA$ and $5 \%$ in the range $[4,7] \AA$. For all experiments, the incident beam divergence was $\sim 0.2 \times 0.2^{\circ}$ (horizontal $\times$ vertical), which gives an average $\gamma$ value of $\sim 0.2^{\circ}=9.5^{\prime}$.

ENGIN-X has a pixelated transmission detector, composed of a $10 \times 10$ array of $2 \times 2 \mathrm{~mm}$ scintillating detectors (glass GS20), arranged on a $2.5 \mathrm{~mm}$ pitch (Santisteban et al., 2002). So, a TOF spectrum similar to that shown in Fig. 3 is measured by each pixel. For experiments not requiring spatial resolution the pixels are summed together for a faster count rate.

In the present work, the least-squares refinements were performed with the parameter $\tau_{h k l}(\lambda)$, describing the exponential decay associated with the moderator contribution to the peak width, kept fixed. The dependence of $\tau$ on neutron wavelength was measured by conventional diffraction experiments on a reference $\mathrm{CeO}_{2}$ powder using ENGIN-X diffraction banks (Santisteban et al., 2006).

\subsection{Cu monochromator}

$\mathrm{Cu}$ mosaic crystals are used in neutron spectrometers as monochromators to select specific neutron wavelengths. The

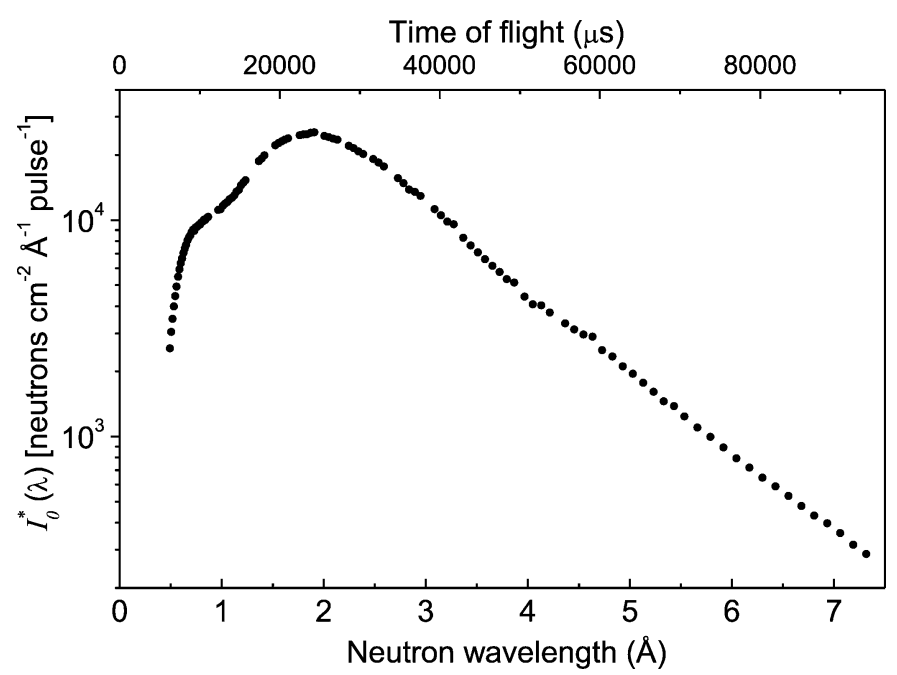

Figure 3

Incident polychromatic neutron spectrum of ENGIN-X, as a function of the neutron time of flight and the neutron wavelength. diffracted intensity from a neutron monochromator is roughly proportional to its mosaicity. Monochromators are usually required to display an anisotropic mosaic spread, with the mosaicity in the diffraction plane matching the incident beam divergence, whilst normal to this plane the mosaicity is kept low for efficient focusing of the beam on the sample. However, $\mathrm{Cu}$ crystals are grown rather perfectly, with mosaicities of a few minutes of arc, making them useless as neutron monochromators. So, several methods are used in order to increase the mosaic spread of perfect crystals through elastic bending or plastic deformation. The production of mosaic crystals with a controlled and homogeneous mosaicity by plastic deformation is not an easy task, and so far the process is not very efficient: a proportion of the crystals are unusable at the end of the production stage and a significant amount of time is spent in testing and characterization (Courtois et al., 2006).
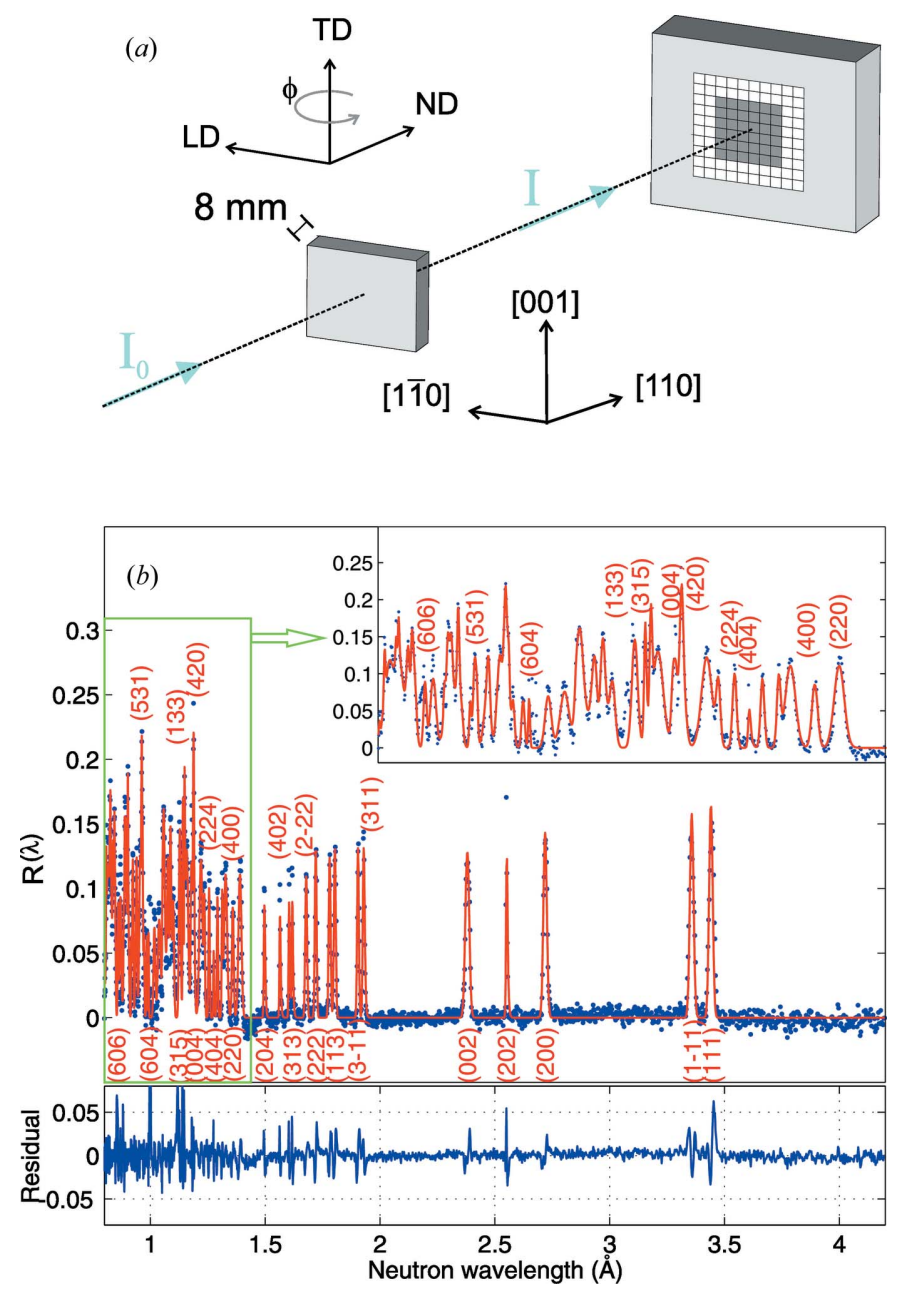

Figure 4

(a) Experimental arrangement for the transmission experiment on the $\mathrm{Cu}$ mosaic crystal specimen at room temperature. The specimen was rotated around the vertical TD direction, and transmission spectra were acquired for rotation angles $\phi$ of $0,2.5,5$ and $10^{\circ}$. Transmission spectra were produced by adding together the central 36 pixels of the detector bank (identified in grey in the detector shown in the figure). (b) The Braggreflected component obtained from the experimental spectrum measured at $\phi=0^{\circ}$, after subtraction of a five-degree polynomial baseline (blue dots), together with the full-profile refinement (solid red line) 
Table 1

Room-temperature lattice parameters, mosaicity and direction cosines obtained from the full-pattern analysis for the three samples investigated in this work.

The table reports the values of lattice parameter and mosaicity refined from the spectra taken for different orientations of the specimens, and the resulting average value.

\begin{tabular}{|c|c|c|c|c|c|c|c|c|}
\hline \multirow[b]{2}{*}{ Sample } & \multirow{2}{*}{\multicolumn{2}{|c|}{ Lattice parameter $(\AA)$}} & \multirow[b]{2}{*}{ Mosaicity $(')$} & \multicolumn{4}{|c|}{ Direction cosines } & \multirow[b]{2}{*}{$G(\%)$} \\
\hline & & & & $a_{11}$ & $a_{12}$ & $a_{13}$ & $\phi_{\exp }\left({ }^{\circ}\right)$ & \\
\hline \multirow{5}{*}{$\begin{array}{l}\text { Copper } \\
\text { monochromator }\end{array}$} & Average & $3.6144 \pm 0.0007$ & $3.0 \pm 0.1$ & - & - & - & - & - \\
\hline & $\phi=0^{\circ}$ & 3.6148 & 3.12 & 0.7524 & 0.0183 & 0.6304 & - & 4.3 \\
\hline & $\phi=2.5^{\circ}$ & 3.6151 & 2.92 & 0.7514 & 0.0620 & 0.5581 & 2.49 & 4.9 \\
\hline & $\phi=5^{\circ}$ & 3.6137 & 3.05 & 0.7491 & 0.1046 & 0.4792 & 4.95 & 4.2 \\
\hline & $\phi=10^{\circ}$ & 3.6140 & 2.95 & 0.7400 & 0.1909 & 0.2658 & 9.98 & 4.3 \\
\hline \multirow{5}{*}{$\begin{array}{l}\text { Sikhote-Alin } \\
\text { meteorite }\end{array}$} & Average & $2.8626 \pm 0.0005$ & $40 \pm 2$ & - & - & - & - & - \\
\hline & $\phi=0^{\circ}$ & 2.8621 & 39.5 & 0.6979 & 0.7084 & 0.0110 & - & 7.7 \\
\hline & $\phi=2^{\circ}$ & 2.8625 & 38.3 & 0.6843 & 0.7149 & 0.0206 & 1.79 & 7.8 \\
\hline & $\phi=4^{\circ}$ & 2.8632 & 40.5 & 0.6628 & 0.7358 & 0.0193 & 3.91 & 10.9 \\
\hline & $\phi=8^{\circ}$ & 2.8626 & 42.4 & 0.6083 & 0.7686 & 0.0434 & 7.84 & 7.6 \\
\hline \multirow[t]{5}{*}{ Pyrite crystal } & Average & $5.4155 \pm 0.0003$ & $26 \pm 2$ & - & - & - & - & - \\
\hline & $\phi=0^{\circ}$ & 5.4154 & 24.1 & 0.9999 & 0.0149 & 0.0043 & - & 6.3 \\
\hline & $\phi=2.5^{\circ}$ & 5.4157 & 28.3 & 0.9988 & 0.0145 & 0.0464 & 2.42 & 10.4 \\
\hline & $\phi=5^{\circ}$ & 5.4152 & 25.1 & 0.9959 & 0.0148 & 0.0892 & 4.85 & 9.90 \\
\hline & $\phi=10^{\circ}$ & 5.4158 & 26.5 & 0.9847 & 0.1351 & 0.1097 & 9.76 & 13.5 \\
\hline
\end{tabular}

Table 2

Refined and derived peak parameters for the $\mathrm{Cu}$ monochromator measured at room temperature and $\phi=0^{\circ}$.

\begin{tabular}{rrllllll}
\hline$h$ & \multicolumn{1}{c}{$k$} & $l$ & $\lambda_{h k l}(\AA)$ & $y_{h k l}$ & $\varpi_{h k l}(\AA)$ & $\mathcal{M}_{h k l}$ & $1 / y_{h k l}$ \\
\hline 1 & 1 & 1 & 3.442 & 0.004 & 0.020 & 248.453 & 242.842 \\
1 & -1 & 1 & 3.358 & 0.004 & 0.021 & 233.918 & 239.571 \\
2 & 0 & 0 & 2.720 & 0.007 & 0.019 & 140.058 & 135.350 \\
2 & 0 & 2 & 2.550 & 0.006 & 0.008 & 145.458 & 160.988 \\
0 & 0 & 2 & 2.380 & 0.010 & 0.021 & 108.353 & 104.173 \\
3 & 1 & 1 & 1.928 & 0.020 & 0.010 & 51.983 & 49.981 \\
3 & -1 & 1 & 1.905 & 0.020 & 0.010 & 49.987 & 50.347 \\
1 & 1 & 3 & 1.804 & 0.024 & 0.011 & 41.937 & 41.531 \\
1 & -1 & 3 & 1.781 & 0.024 & 0.011 & 39.906 & 41.914 \\
2 & 2 & 2 & 1.721 & 0.028 & 0.011 & 36.000 & 35.954 \\
2 & -2 & 2 & 1.679 & 0.025 & 0.011 & 33.341 & 39.471 \\
3 & 1 & 3 & 1.617 & 0.023 & 0.006 & 31.822 & 44.138 \\
3 & -1 & 3 & 1.604 & 0.023 & 0.006 & 31.254 & 43.605 \\
4 & 0 & 2 & 1.564 & 0.022 & 0.006 & 24.439 & 44.910 \\
2 & 0 & 4 & 1.496 & 0.031 & 0.007 & 21.582 & 32.484 \\
2 & 2 & 0 & 1.391 & 0.039 & 0.016 & 26.578 & 25.814 \\
4 & 0 & 0 & 1.360 & 0.039 & 0.010 & 18.482 & 25.807 \\
\hline
\end{tabular}

We have made test experiments on a small as-grown $\mathrm{Cu}$ monochromator, $30 \mathrm{~mm}$ long, $30 \mathrm{~mm}$ wide and $8 \mathrm{~mm}$ thick, with the [110] crystallographic direction along the normal direction, produced by the Optics Laboratory of the Institut Laue-Langevin, France. The reported mosaicity of the specimen is $3^{\prime}$, as measured with a polychromatic $\gamma$-ray diffractometer (Bastie et al., 2000).

The $\mathrm{Cu}$ mosaic crystal specimen was investigated at room temperature $(\sim 293 \mathrm{~K})$ using the experimental arrangement shown in Fig. 4(a). The specimen was rotated around the vertical TD direction, and transmission spectra were acquired for rotation angles $\phi$ of $0,2.5,5$ and $10^{\circ}$, with counting times of 15 min per orientation. Transmission spectra were produced by adding together the central 36 pixels of the detector bank (identified in grey in the detector shown in the figure). This corresponds to a sampling area of $14 \times 14 \mathrm{~mm}$ and an effective detector area of $36 \times 4=144 \mathrm{~mm}^{2}$. The blue dots in Fig. 4(b) show the Bragg-reflected component $R(\lambda)$ obtained from the experimental $[1-T(\lambda)]$ spectrum measured at $\phi=$ $0^{\circ}$, after subtraction of a five-degree polynomial baseline.

All $R(\lambda)$ spectra measured here were leastsquares refined using the full-pattern analysis described in the previous section. The red solid line in Fig. 4(b) shows the result of such refinement for the $\mathrm{Cu}$ monochromator measured at room temperature and $\phi=0^{\circ}$, where most peaks of the face-centred cubic (f.c.c.) crystal structure have been clearly indexed. The fitting is very good, as can be appreciated from the difference curve shown at the bottom and the detail of the shortwavelength range displayed in the inset. Moreover, the $G$ parameter obtained in this refinement $(4.3 \%)$ is of the same order of magnitude as the statistical uncertainty of the measurement, which is a direct manifestation of a high-quality refinement. The values of the lattice parameter, mosaicity and direction cosines measured for each orientation are listed in Table 1, together with the $G$ parameter obtained in each case. The average refined values for the lattice parameter and the crystal mosaicity were $a=3.6144 \pm 0.0007 \AA$ and $\eta=3.0 \pm 0.1^{\prime}$, where the uncertainties correspond to the standard deviation of the parameters refined from the spectra taken at the different orientations. The very good agreement between the refined values and the lattice parameter reported in the literature (3.613 А; Gale \& Totemeier, 2004) and the crystal mosaicity measured by another technique indicates the efficiency of the proposed method to characterize $\mathrm{Cu}$ crystals.

Also listed in the table are the rotation angles $\phi$ of the incident neutron beam, as seen from the coordinate system of the crystal, calculated from the direction cosines refined for each orientation and those obtained at $\phi=0^{\circ}$. The resulting values $\left(2.49,4.95\right.$ and $9.98^{\circ}$, respectively) agree very well with those given by the goniometer control system. This demonstrates the angular resolution $\left(\sim 1^{\prime}\right)$ achievable by the proposed method to define crystal orientations. Moreover, by applying the method described in $\$ 3.4 .5$ to the spectra recorded at 0 and $2.5^{\circ}$, we have found that the [001] and the [1]̄0] directions of the crystal are $3.41^{\circ}$ off the transverse and longitudinal directions of the specimen, respectively.

The extinction factors obtained for the $\mathrm{Cu}$ monochromator at room temperature are listed in Table 2 and will be analysed in a later section, together with the results obtained for the other specimens.

Following the room-temperature experiments, transmission spectra were recorded for temperatures between 55 and $225 \mathrm{~K}$, with the $\mathrm{Cu}$ monochromator oriented with its [110] direction making an angle of $\sim 30^{\circ}$ with the incident beam and the [1] 0 ] direction pointing vertically. The specimen was placed in an Oxford Instruments Variox Cryostat and 
measurements were performed at 55, 70, 85, 135, 190 and $225 \mathrm{~K}$, with counting times of $12 \mathrm{~min}$ per temperature. No rotations were applied to the specimen for this case. Fig. 5 shows the $(1-T)$ spectra measured at 55 and $225 \mathrm{~K}$, obtained by adding the counts registered by the central five-pixel column of the transmission detector. As clearly observed in Fig. 5, the scattering component of the attenuation coefficient of the monochromator increases with temperature. The continuous grey and red lines shown as baselines of the two experimental spectra are not polynomial fits but theoretical calculations of $A(\lambda)+S(\lambda)$ using equations (6), (7) and (8), for a traversed specimen thickness of $16 \mathrm{~mm}$ and a Debye temperature of $313 \mathrm{~K}$. The very good agreement between experiment and calculation validates the model used for the scattering component $S(\lambda)$, described in detail in Appendix $A$. Besides the change in $S(\lambda)$, the increase in temperature slightly changes the intensity and position of the peaks of the Bragg-reflected component. Fig. 6 shows the thermal expansion of the $\mathrm{Cu}$ lattice parameter determined from the fullpattern refinement of the cryostatic experiments. The analysis used the 18 peaks contained within the $[1.4,4.2]$ Å wavelength interval. For each temperature, the analysis was performed on the spectra produced not only for the central column shown in the figure but also for four other columns, two at each side of the central one. So, in this case the error bands displayed in Fig. 6 correspond to the standard deviation of the lattice parameter refined from the five spectra recorded at each temperature, which represent the spatial variation found across the specimen. Good agreement is observed between the present experiments and previous measurements of lowtemperature lattice parameters measured on pure $\mathrm{Cu}$ using a rotating single-crystal back-reflection X-ray diffraction method, also shown in Fig. 6 (Simmons \& Balluffi, 1957). On the other hand, the refined values for the mosaicity exhibited a nearly constant dependence on temperature, indicating that

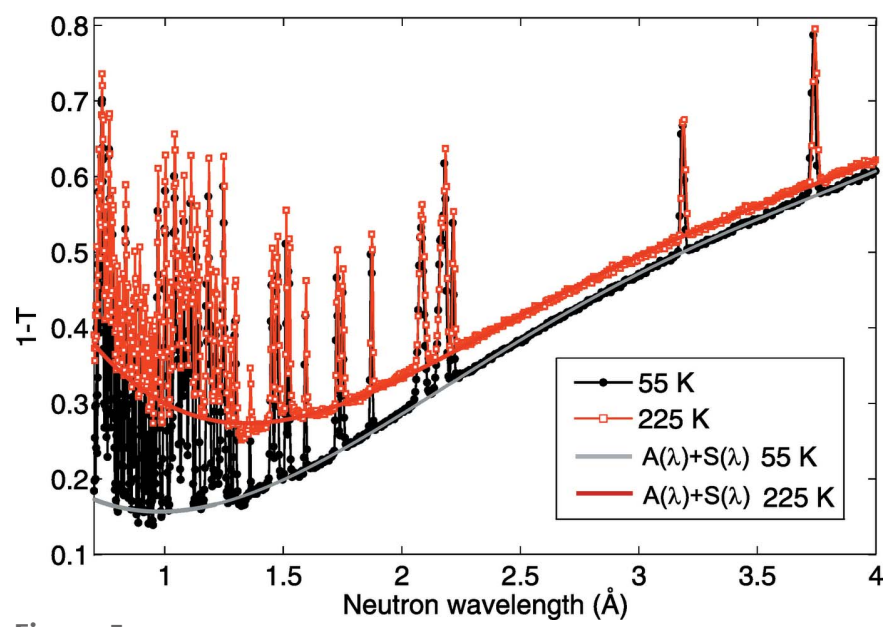

Figure 5

The $(1-T)$ spectra measured at $55 \mathrm{~K}$ (black dots) and $225 \mathrm{~K}$ (red squares), obtained by adding the counts registered by the central fivepixel column of the transmission detector. The continuous grey and red lines shown as baselines of the two experimental spectra are not polynomial fits but theoretical calculations of $A(\lambda)+S(\lambda)$ for an effective specimen thickness of $16 \mathrm{~mm}$ and a Debye temperature of $313 \mathrm{~K}$. the mosaicity of the specimen remains the same throughout the experiment.

\subsection{Iron-nickel meteorite}

The full-pattern analysis was applied to transmission measurements performed on an iron-nickel meteorite from the Sikhote-Alin fall. In this fall, a meteorite of an estimated mass of 1000 tonnes exploded at an altitude of $6000 \mathrm{~m}$ on the Sikhote-Alin Mountains, in south-eastern Russia, in 1947 (Buchwald, 1976). Thousands of fragments of the meteorite were collected, varying from less than $1 \mathrm{~g}$ to $1700 \mathrm{~kg}$. Specimens from this fall are of two types. Those commonly called 'complete individuals', showing ablation and a fusion crust, are probably the ones that broke off from the main mass early in the decent. They are characterized by regmaglypts on their surface, i.e. ablation cavities resembling thumbprints. The second type of Sikhote-Alin specimens are 'fragments', which show the effects of being torn apart from the explosion or on impact with the ground. They look like shrapnel from violent explosions (Gallant, 1996). Both types of meteorites are composed mostly of kamacite [body-centred cubic (b.c.c.) $\mathrm{FeNi}$ ] with a small quantity of taenite (f.c.c. NiFe). Generally, most of the meteoritic metal cooled very slowly from temperatures within the single-phase taenite field and a Widmanstätten structure consisting of kamacite plates aligned along the octahedral planes of the taenite matrix was produced during cooling (Reisener \& Goldstein, 2003). Depending on the nickel content, the lattice parameters of the taenite and kamacite vary slightly. The measured lattice parameters for taenite and pure f.c.c. iron-nickel alloys are in the range of about $3.51-3.60 \AA$ and those for kamacite and b.c.c. iron-nickel fall between 2.86 and $2.89 \AA$ (He et al., 2006).

Previous transmission experiments on a $\sim 10 \mathrm{~mm}$-thick complete individual with regmaglypts showed that the

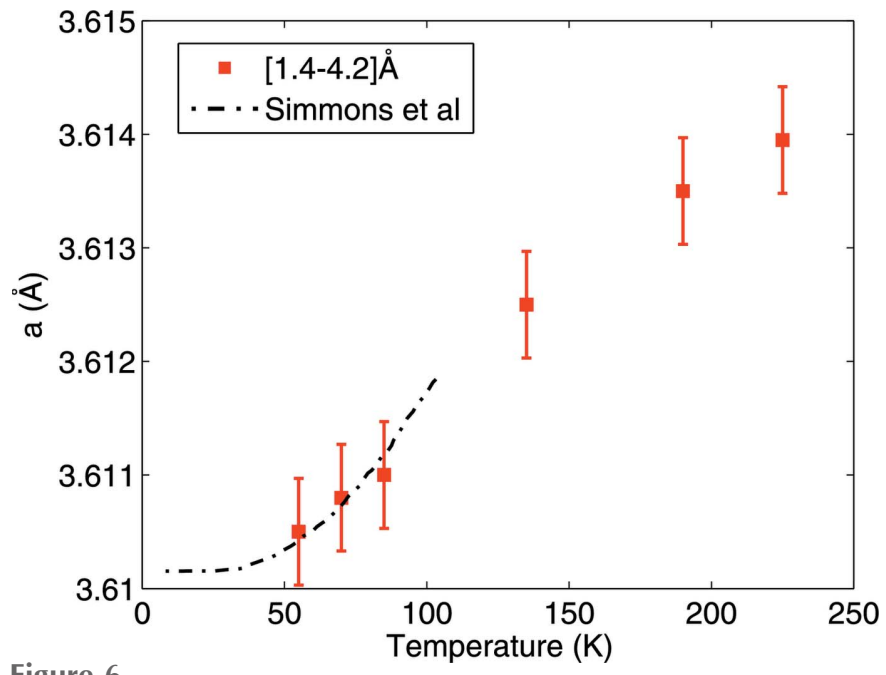

Figure 6

Thermal expansion of the $\mathrm{Cu}$ lattice parameter determined from the fullpattern refinement of the cryostatic experiments (red squares). The error bars correspond to the standard deviation of the lattice parameter refined from the five spectra recorded at each temperature. Good agreement is observed between the present experiments and previous measurements (dash-dot black line). 
specimen is essentially a single crystal (Santisteban, 2005). Here, we have measured the transmission of the same specimen but at different orientations, using the experimental arrangement shown in Fig. 7(a). Transmission spectra were recorded for four orientations, by rotating the specimen around the vertical direction, identified as the $x_{1}$ axis in the figure. The rotation angles were $\phi=0,2,4$ and $8^{\circ}$, with counting times of $10 \mathrm{~min}$ per orientation. For each orientation, a transmission spectrum was produced by adding together the central $4 \times 4=16$ pixels of the pixelated detector, which corresponds to a sampling area of $9.5 \times 9.5 \mathrm{~mm}$ and an effective detector area of $8 \times 8 \mathrm{~mm}$.

Fig. 7(b) shows in blue dots the experimental $R(\lambda)$ measured at $\phi=0^{\circ}$, together with the full-pattern refinement using a single kamacite crystal (red solid line). The values of the lattice parameter, mosaicity and direction cosines measured for each orientation are listed in Table 1. The average refined values for the lattice parameter and the crystal mosaicity are $a=2.8626 \pm 0.0005 \AA$ and $\eta=40 \pm 2^{\prime}$, where the uncertainties correspond to the standard deviation of the parameters refined from the spectra taken at the different orientations. The refined lattice parameter for the kamacite phase is in an excellent agreement with the values found by other authors (Owen et al., 1937; McKeehan, 1923) and corresponds to an average of $5 \%$ nickel in solid solution
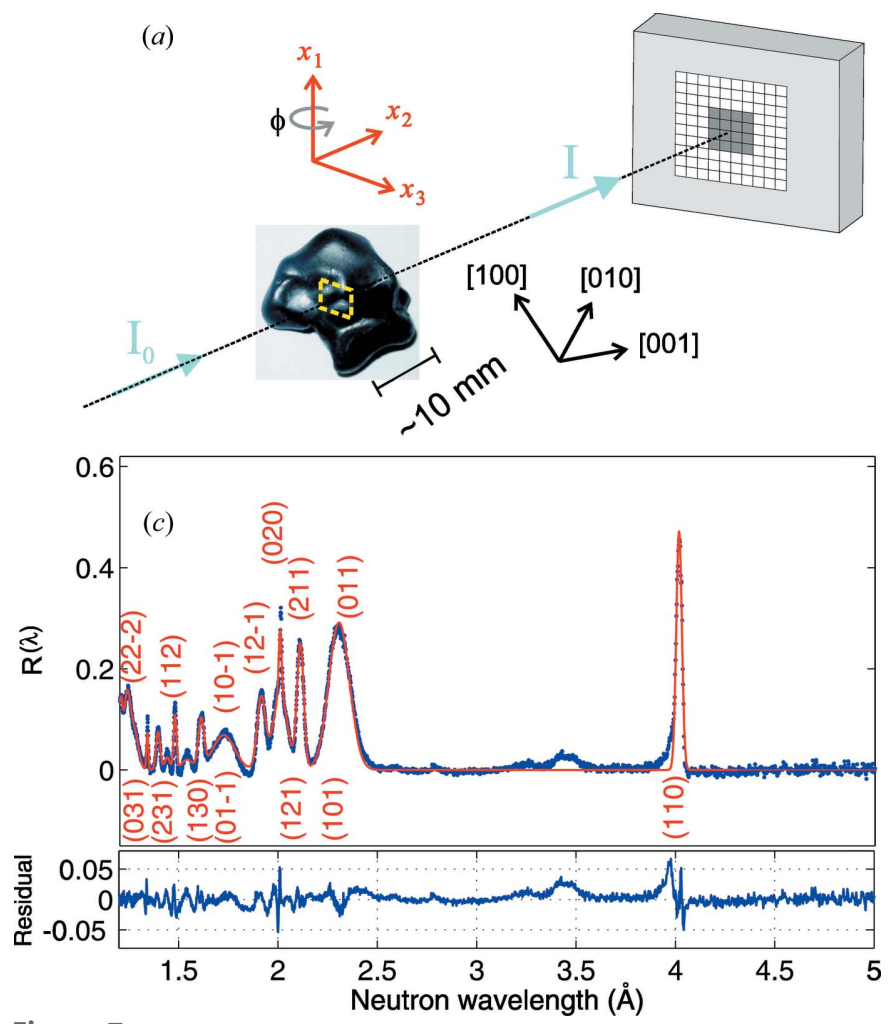

Figure 7

(a) Experimental arrangement for the transmission experiment on the $\mathrm{NiFe}$ meteorite (picture). Transmission spectra were recorded for four orientations $\left(\phi=0,2,4\right.$ and $\left.8^{\circ}\right)$ by rotating the specimen around the vertical direction, identified as the $x_{1}$ axis in the figure. $(b)$ The Braggreflected component obtained from the experimental spectrum measured at $\phi=0^{\circ}$ (blue dots), together with the full-profile refinement (solid red line) using a single kamacite crystal.
(Owen \& Burns, 1939). On the other hand, the relatively large value found for the mosaicity could be caused by local changes in orientation of a large kamacite crystal, over the macroscopic volume sampled by the experiment. However, this large value of mosaicity is observed even at the pixel level, so the misorientation of the crystal blocks must occur across the neutron beam direction.

Although the overall full-pattern refinement is rather good, with $G$ parameters between 7.7 and $10.9 \%$, a clear difference with the experimental data emerges in the $[3,4] \AA$ 皮 wavelength interval, as is shown in the residual plot at the bottom of Fig. 7(b). Such discrepancy reveals the presence of a small quantity of material of different microstructure, which could be either one or more kamacite or taenite crystals of different orientation from the main crystal, or even a polycrystalline region contained within the relatively large volume gauged by the experiment.

\subsection{Pyrite mineral}

A series of neutron transmission experiments were performed on a natural iron pyrite mineral, $\mathrm{FeS}_{2}$, the most abundant of all sulfide minerals associated with the Earth's surface region. Pyrite belongs to the family of $M X_{2}(M$ : transition atom; $X$ : chalcogenide atom) type compounds with the same crystal structure, and the name 'pyrite' is often used to represent all the crystals belonging to that family. The diverse electric and magnetic properties and their interplay in pyrite type compounds have attracted much interest. In particular, the structural, optical, electronic and other properties of natural iron pyrite have been investigated by solidstate physicists and chemists for many years (Sato, 1985; Birkholz, 1992; Antonijevic et al., 2002). More recently, owing to their excellent light absorbing and electronic properties, the pyrite compounds have attracted an enormous amount of research attention in the field of photovoltaic materials (Schlegel \& Wachter, 1976; Schieck et al., 1990), and the possible relation of the physical properties to chemical composition has been investigated by several authors (Ferrer \& Sánchez, 1992; Echarri \& Sánchez, 1974).

Pyrite crystallizes in a rock salt type structure (f.c.c.) and belongs to the space group $P a 3$, with the unit cell containing four $\mathrm{FeS}_{2}$ formula units. The mineral adopts a cubic $\mathrm{NaCl}$-like structure with the $\mathrm{Fe}$ atoms on the corners and face centre positions of the unit cell and the $S_{2}$ units lying at the midpoints of the 12 edges and in the centre of the cube. Therefore, the unit cell is completely defined by the lattice parameter $a$ and the $\mathrm{S}$ coefficient $u$, which defines the coordinates of each $\mathrm{S}$ atom in the unit cell. The crystal structure of pyrite was first published in 1914 (Bragg, 1914), and the defining parameters are $a=5.416 \AA$ and $u=0.385 \AA$ (Murphy \& Strongin, 2009). Regarding the pyrite morphology, natural crystals can exhibit a wide variety of habits. However, most of them are defined by the combination of a reduced number of forms, mainly cubic, octahedral and pyritohedral form, where the morphology is determined by the temperature and degree of the solution supersaturation (Murowchick \& Barnes, 1987). In particular, 
single-crystal cubes tend to grow under low temperatures from solutions with low concentrations of iron and sulfur. However, owing to variations in natural conditions, the final macroscopic crystals exhibit some degree of misorientation between the single-crystal blocks, i.e. the crystal exhibits a mosaic structure. Here we study the mosaic structure of a natural cubic iron pyrite by applying the full-profile refinement analysis to the transmission spectra recorded at different sample orientations.

The geometry and orientation of the pyrite specimen investigated are shown in Fig. 8(a), together with a photograph of the specimen, a nearly perfect cube of $15 \mathrm{~mm}$ side. To aid with the presentation of the results, a macroscopic coordinate system was fixed to the specimen, as shown in the figure. As in the previous cases, several transmission spectra were collected at different orientations of the specimen, obtained by rotating the crystal around the vertical direction (TD in the sample coordinate system). The investigated angles were $\phi=0,2.5,5$ and $10^{\circ}$, with counting times of $12 \mathrm{~min}$ per orientation. The angle $\phi=0^{\circ}$ corresponds to an orientation in which ND was aligned with the incident beam.
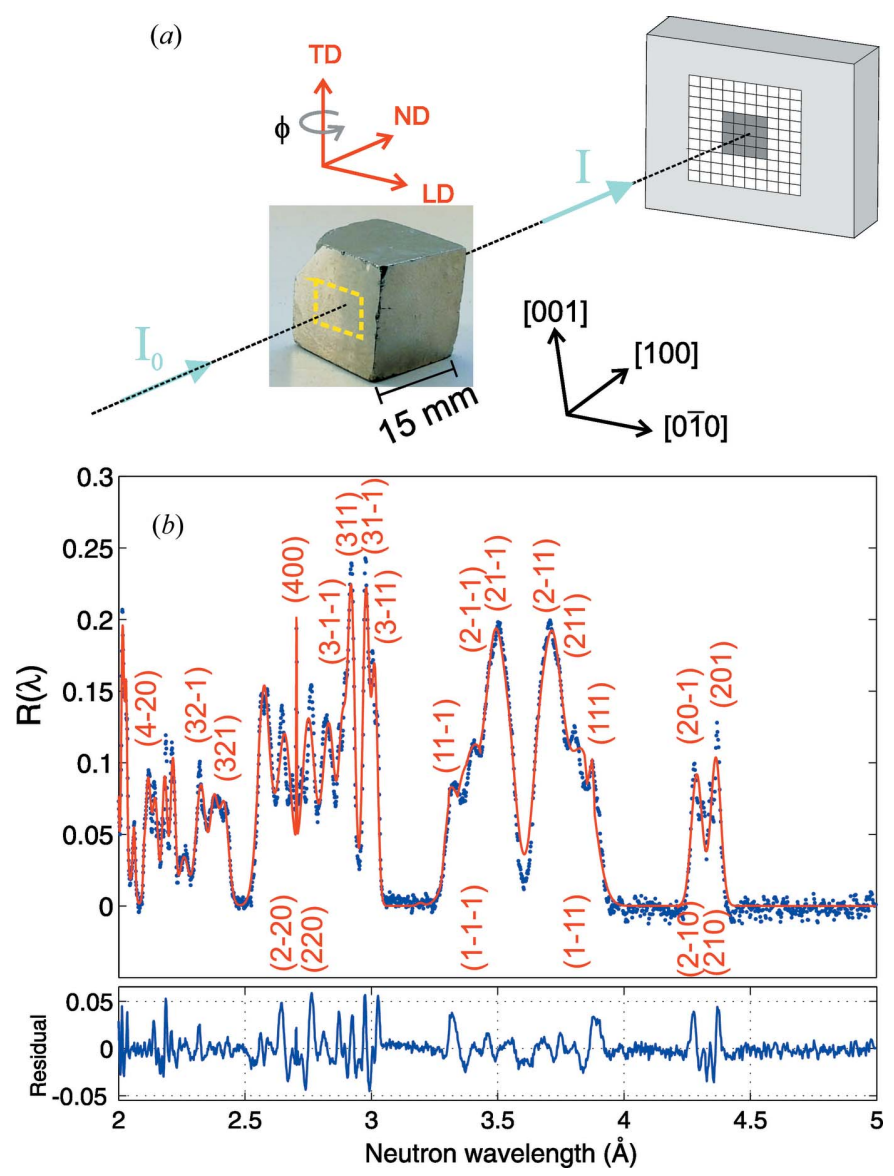

Figure 8

(a) Experimental arrangement for the transmission experiment on the natural pyrite specimen (picture). Transmission spectra were recorded for four orientations $\left(\phi=0,2.5,5\right.$ and $\left.10^{\circ}\right)$ by rotating the specimen around TD. (b) The Bragg-reflected component obtained from the experimental spectrum measured at $\phi=0^{\circ}$ (blue dots), adding together the central 16 pixels of the pixelated detector [identified in grey in the detector shown in (a)]. The results of the full-pattern least-squares refinement performed within a $[2,5] \AA$ wavelength range are shown as a solid red line.
As in the previous case, a transmission spectrum was produced for each orientation, by adding together the central $4 \times 4=16$ pixels of the pixelated detector, identified in Fig. $8(a)$. Fig. $8(b)$ shows the experimental $R(\lambda)$ spectrum recorded at the orientation $\phi=2.5^{\circ}$, together with the results of the full-pattern least-squares refinement performed within the $[2,5] \AA$ wavelength range. The least-squares refinement was performed with the parameter $u$ (which defines the position of the $\mathrm{S}$ atoms) fixed to a value of $0.385 \AA$. The values of the lattice parameter, mosaicity and direction cosines measured for each orientation are listed in Table 1. The average refined values for the lattice parameter and crystal mosaicity are $a=5.4155 \pm 0.0003 \AA$ and $\eta=26 \pm 2^{\prime}$, respectively. As before, the reported uncertainties correspond to the standard deviation of the parameters refined from the spectra taken at the different orientations. An excellent agreement was found between the average lattice parameter and the value reported in the literature $(5.416 \AA$; Murphy \& Strongin, 2009). Also listed in Table 1 are the rotation angles $\phi$ of the incident neutron beam, as seen from the coordinate system of the crystal. Comparison between the refined and nominal values suggests a difference of $0.08^{\circ}$ between the

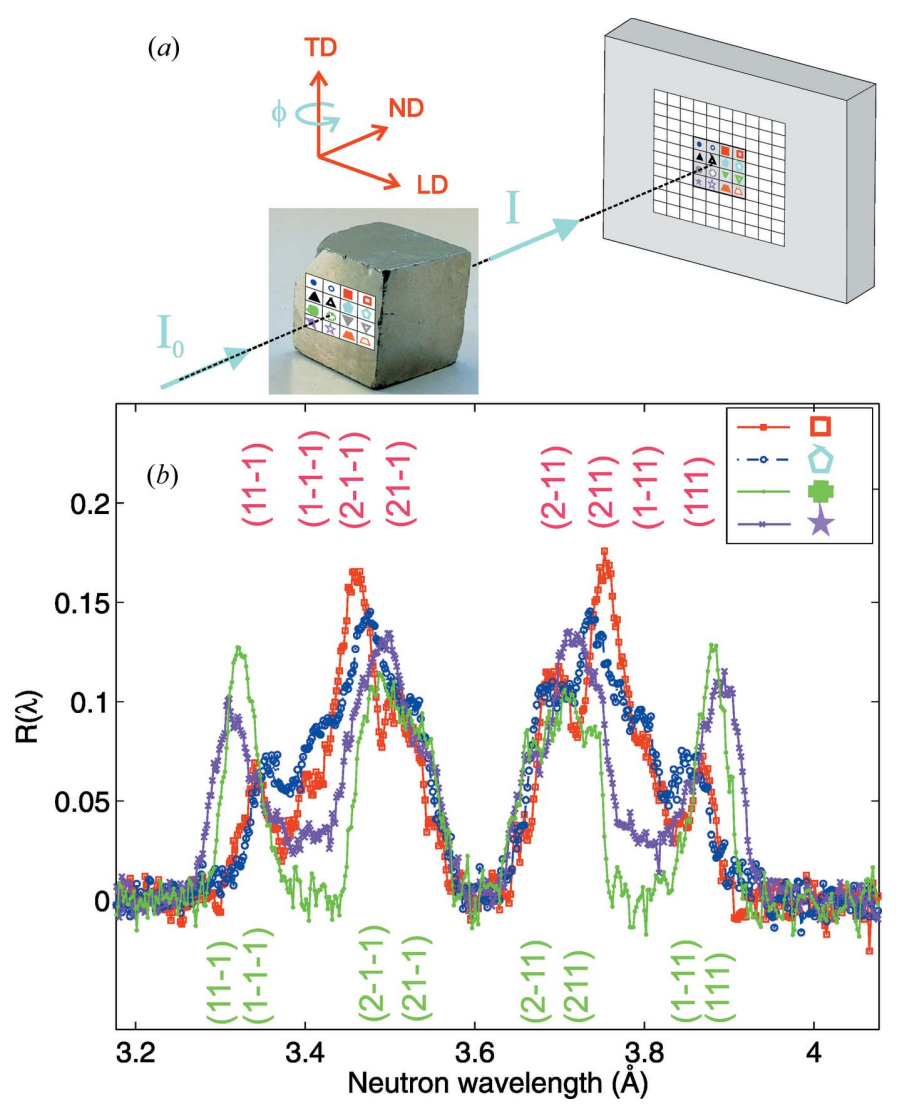

Figure 9

(a) Experimental configuration for the spatially resolved transmission experiment on the pyrite sample. An individual transmission spectrum was obtained for each of the 16 pixels, as schematically identified by different symbols in the figure. (b) A detail of the $R(\lambda)$ spectra recorded by four pixels for the $\phi=0^{\circ}$ orientation. Clear differences are observed between the different spectra, with the peaks from the $\{211\}$ family shifting positions, revealing misorientations between the different regions gauged by each pixel. 
requested angles and those actually achieved by the goniometer.

Finally, we have calculated the overall transformation matrix between the crystal system and the macroscopic system attached to the specimen, as schematically shown in Fig. 8. The differences between characteristic crystal directions and the macroscopic sample directions are $\beta_{1}=0.88^{\circ}, \beta_{2}=1.08^{\circ}$ and $\beta_{3}=0.57^{\circ}$, where $\beta_{1}$ is the angle between the [100] crystal direction and ND, $\beta_{2}$ is the angle between the [010] crystal direction and $\mathrm{LD}$, and $\beta_{3}$ is the angle between the [001] crystal direction and TD. This corresponds in Euler angles to $\phi_{1}=312.5^{\circ}, \Phi=81.7^{\circ}$ and $\varphi_{2}=1.5^{\circ}$, where the Bunge convention was applied to define the rotation axis order.

Naturally occurring materials are usually inhomogeneous. So, after analysis of the overall transmission of the sample, we performed a spatially resolved analysis aimed at assessing the variation of the reported parameters across the specimen. For this purpose, we produced an individual transmission spectrum for each of the 16 pixels, as schematically identified by different symbols in Fig. 9(a). Fig. 9(b) shows a detail of the $R(\lambda)$ spectra recorded by four pixels for the $\phi=0^{\circ}$ orientation. Clear differences are observed between the different spectra, with the peaks from the $\{211\}$ family shifting positions, revealing misorientations between the different regions gauged by each pixel. Besides this, the smaller width observed for the pixel denoted by a green cross points to a smaller mosaicity. In order to quantify these observations, full-pattern refinements were performed for the $16 R(\lambda)$ spectra recorded at the $\phi=0^{\circ}$ and $\phi=2.5^{\circ}$ specimen orientations. The direction cosines of the neutron beam in the crystal system for each pixel and sample orientation were obtained from full-pattern refinements of the experimental data. The position-dependent orientation matrix of the crystal for each pixel was calculated using the method described in $\$ 3.4 .5$. The crystal orientations for the 16 locations of the pyrite crystal were expressed in terms of the three Euler angles $\left(\varphi_{1}^{\mathrm{C}}, \Phi^{\mathrm{C}}, \varphi_{2}^{\mathrm{C}}\right)$ within the threedimensional Euler space. The points cluster into two different regions centred at $\left(332^{\circ}, 28^{\circ}, 1^{\circ}\right)$ and $\left(271^{\circ}, 90^{\circ}, 2^{\circ}\right)$, indicating

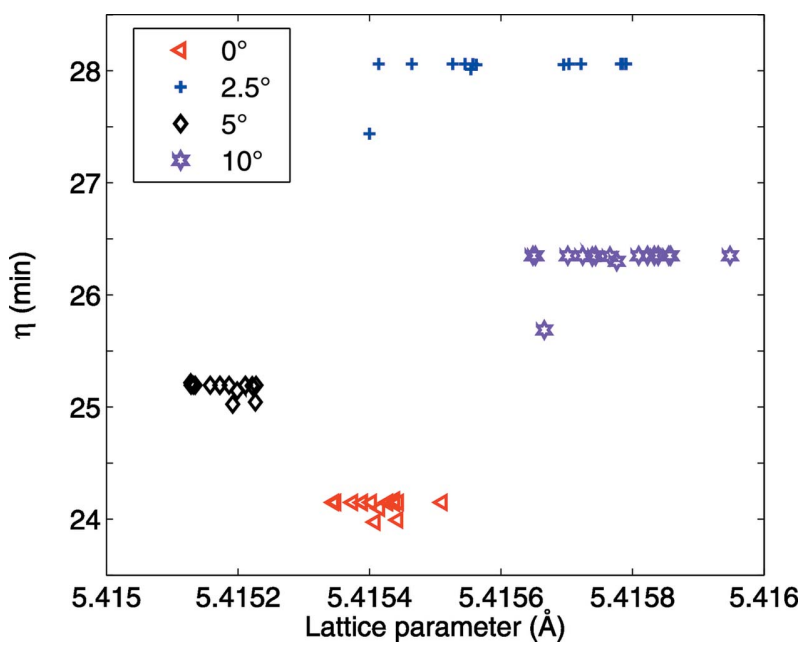

Figure 10

The refined values for the lattice parameter and crystal mosaicity for each pixel at different sample orientation. that the specimen is in fact composed of at least two regions, slightly misaligned by about $2^{\circ}$ around an axis along the [0 $\left.\overline{1} 0\right]$ direction. The correlation between the refined values for the lattice parameter and crystal mosaicity for each one of the 16 pixels at different sample orientation is shown in Fig. 10. As seen in the figure, the refined lattice parameter for all sample rotations exhibits a very small variation $(\sim 0.01 \%)$ across the specimen (note the scale of the $x$ axis), whilst the crystal mosaicity exhibits variations of $\sim 10 \%$ between the different orientations. This could be a consequence of the inhomogeneity of the sample. As the specimen is rotated, the average misorientation of the crystal blocks traversed by the neutron beam varies, which is reflected as slightly different crystal mosaicities obtained in the full-pattern refinement.

\section{Discussion}

Figs. $4(b), 7(b)$ and $8(b)$ have shown that the experimental fraction of Bragg-reflected neutrons $R(\lambda)$ is very well described by the full-pattern model presented in equation (11). Moreover, the least-squares refinement implementation of this model described in $\$ 3$ has proven very efficient and has produced sound values of lattice parameters, mosaicities and crystal orientation as summarized in Table 1 . Note that the refined values show little dependence on the orientation of the specimen in the beam, which highlights the robustness of the technique. In fact, we have adopted here the standard deviation of the results obtained at different specimen orientation as a measure of the uncertainty of the reported values. This estimation is conservative, because by changing the orientation of the specimen we also change the effective volume of the specimen being gauged by the neutron beam. Provided the specimens investigated are inhomogeneous, a part of these differences may actually have a real origin. The reported values of the parameter $G$, indicating the goodness of the refinements, are consistent with these ideas. Better agreement ( $G \simeq 4.5 \%$ ) is found for the man-made and well characterized $\mathrm{Cu}$ monochromator, whilst poorer $G$ values were obtained for the naturally occurring specimens, the iron-nickel meteorite and the pyrite crystal. This becomes more evident by comparing the refinements of Figs. $4(b), 7(b)$ and $8(b)$. For the $\mathrm{Cu}$ monochromator at room temperature (Fig. 4b) the fullpattern analysis shows an excellent agreement with the experimental $R(\lambda)$ pattern, even for the short-wavelength range where the Bragg peaks are highly superimposed. By contrast, the refinement of the iron-nickel meteorite (Fig. 7b) reveals some differences between 3 and $4 \AA$ that suggest the presence of a small fraction of differently oriented crystals (or crystals from a different phase) which are not being taken into account by the model.

Hence, it is clear that the proposed method can be an efficient tool to characterize microscopic properties of a singlecrystal specimen. So far, we have used it to quantify lattice parameters, mosaicities and orientation of mosaic crystal, so in the following we will briefly discuss the best strategies for this and the accuracy that could be expected for the technique. After that, we will discuss the information provided by the 
extinction factors and the prospects of the proposed fullpattern analysis for dealing with specimens containing several crystals.

\subsection{Optimal wavelength range}

As seen in Figs. 4(b), 7(b) and 8(b), for simple structures the $R(\lambda)$ patterns present two distinct regions: (i) a long-wavelength range $(\lambda>1.5 \AA)$ where most Bragg peaks are well isolated, and (ii) a short-wavelength range $(\lambda<1.5 \AA)$ where Bragg peaks are highly superimposed. A least-squares fit over the full wavelength range requires refinement of a large number of fitting parameters (one for each additional peak) and does not necessarily provide more accurate values of the refined lattice parameters, mosaicities or orientation. So, in order to explore the influence of the wavelength range on the fitting accuracy, the full-profile refinement was applied to the experimental $R(\lambda)$ spectrum recorded for the $\mathrm{Cu}$ monochromator room-temperature experiment at $\phi=0^{\circ}$ using different wavelength intervals. The used fitting intervals were $[0.8,1.45] \AA,[1.45,4.5] \AA$ and $[2,4.5] \AA$, which correspond to 70,16 and 5 reflections, respectively. The corresponding $G$ parameters $(6.5,4.1$ and $4.8 \%)$ showed that the quality of the fit is related to the definition of the Bragg peaks rather than to the number of reflections included in the fitting. So, in principle, it should be possible using the $G$ parameter as a figure of merit to define an optimum wavelength interval to perform the least-squares refinement, whilst still providing the fitting parameters of interest with the requested uncertainty. Of course, the precise wavelength range will depend on the particular material and specimen orientation investigated. For example, for the $\mathrm{Cu}$ monochromator at room temperature we have found that the optimal wavelength interval to perform the fitting is $\sim[1.5,4.5] \AA$. Using this particular wavelength range, the average $G$ parameter obtained from the different orientations is smaller $(\langle G\rangle \simeq 4.2 \%)$ than the values listed in Table 1 obtained using the $[0.8,4.5] \AA$ interval, yet the refined lattice parameter and mosaicity have equivalent uncertainties.

\subsection{Accuracy in lattice parameter}

The reported uncertainty for the measured lattice parameters resulted from the standard deviation of the values refined from the transmission spectra recorded at different orientations of the specimen. Another measure of the uncertainty of the proposed analysis may be obtained by comparing the values of lattice parameters that result from the individual peak positions $\left(\lambda_{h k l}^{\mathrm{sp}}\right)$ and the full-pattern-refined direction cosines:

$$
a=\frac{\lambda_{h k l}^{\mathrm{sp}}}{2} \frac{h^{2}+k^{2}+l^{2}}{\left|h a_{11}+k a_{12}+l a_{13} \cdot\right|} .
$$

The values of $a$ so obtained for the $\mathrm{Cu}$ monochromator at room temperature, calculated for the reflections in the [1.45, 4.5] $\AA$ interval, are displayed in Fig. 11. The standard deviation of these values, $\sigma=0.0008 \AA$, is similar to the uncertainty $\Delta a=0.0007 \AA$ reported for the average $\mathrm{Cu}$ lattice parameter in Table 1. So, we see that the deviation observed in the lattice parameters measured at different orientations is compatible with the accuracy of the model in its description of the observed peak positions. The difference of $\Delta a / a \simeq 300 \mu \varepsilon$ may result from relatively small stresses within the specimen $(-25 \mathrm{MPa})$ which would slightly deviate the peak positions from those expected for a completely unstressed specimen. Provided stresses are anisotropic, different reflections will have different shifts or even opposite signs. Because of this, the refined value of $a$ is expected to change within $\sim 300 \mu \varepsilon$, depending on the number of reflections included in the fullpattern analysis. In principle, the full-pattern model could be improved by incorporating the effect of an arbitrary strain tensor in the peak positions.

On the other hand, Fig. 11 is useful to identify errors in the indexing procedure. A wrongly indexed peak would produce a rather different value of the lattice parameter, which will stand out from the standard deviation of the figure.

\subsection{Extinction factors}

The refined position $\left(\lambda_{h k l}\right)$ (evaluated from the full-pattern model), extinction factor $\left(y_{h k l}\right)$ and FWHM $\left(\varpi_{h k l}\right)$ of the peaks appearing in $R(\lambda)$ for the room-temperature $\mathrm{Cu}$ monochromator, the Sikhote-Alin meteorite and the pyrite crystal are listed in Tables 2, 3 and 4, respectively. The extinction factors $y_{h k l}$ indicate that the measured intensities are much smaller than those expected from the kinematical theory, typically between 0.5 and $25 \%$ of the kinematical value predicted by equation (9). Comparison of the refined extinction factors with reported values is not straightforward because most experimental work on extinction has been carried out using double-crystal arrangements, where a monochromatic neutron beam is incident on the specimen being investigated. The reported extinction factors are highly dependent on the geometry of the experiment, e.g. the divergence and wavelength of the incident beam, as well as on

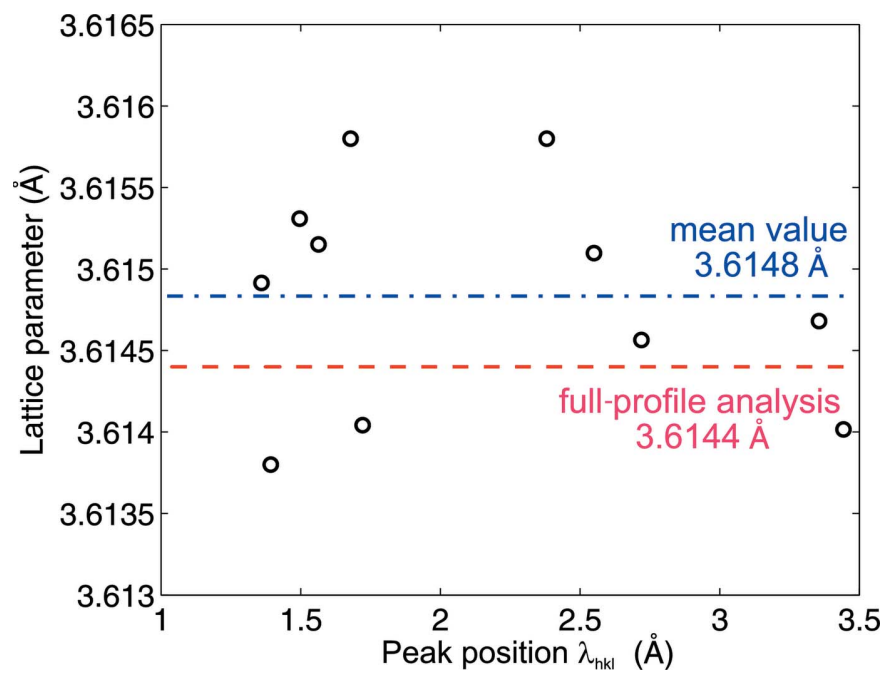

Figure 11

The values of the lattice parameter obtained for the $\mathrm{Cu}$ monochromator at room temperature calculated for the reflections in the $[1.45,4.5] \AA$ interval. 
Table 3

Refined and derived peak parameters for the Sikhote-Alin meteorite at $\phi=0^{\circ}$.

\begin{tabular}{rrrrrrrr}
\hline$h$ & $k$ & $l$ & $\lambda_{h k l}(\AA)$ & $y_{h k l}$ & $\varpi_{h k l}(\AA)$ & \multicolumn{1}{c}{$\mathcal{M}_{h k l}$} & $1 / y_{h k l}$ \\
\hline 1 & 1 & 0 & 4.014 & 0.004 & 0.042 & 282.060 & 249.200 \\
0 & 1 & 1 & 2.322 & 0.019 & 0.033 & 42.640 & 52.540 \\
1 & 0 & 1 & 2.292 & 0.023 & 0.032 & 34.100 & 43.220 \\
1 & 2 & 1 & 2.112 & 0.032 & 0.031 & 33.126 & 30.860 \\
2 & 1 & 1 & 2.102 & 0.043 & 0.031 & 29.174 & 23.157 \\
0 & 2 & 0 & 2.022 & 0.044 & 0.031 & 23.538 & 22.547 \\
2 & 2 & 0 & 2.007 & 0.035 & 0.031 & 23.262 & 28.300 \\
2 & 0 & 0 & 1.992 & 0.038 & 0.031 & 19.950 & 26.149 \\
1 & 2 & -1 & 1.912 & 0.061 & 0.030 & 19.790 & 16.357 \\
2 & 1 & -1 & 1.902 & 0.060 & 0.030 & 18.866 & 16.550 \\
0 & 1 & -1 & 1.722 & 0.042 & 0.029 & 18.144 & 23.972 \\
1 & 0 & -1 & 1.692 & 0.051 & 0.029 & 15.194 & 19.528 \\
1 & 3 & 0 & 1.611 & 0.095 & 0.028 & 13.574 & 10.506 \\
3 & 1 & 0 & 1.600 & 0.039 & 0.028 & 12.850 & 25.351 \\
1 & 1 & 2 & 1.538 & 0.034 & 0.027 & 11.472 & 29.624 \\
2 & 3 & 1 & 1.479 & 0.047 & 0.026 & 10.836 & 21.478 \\
3 & 2 & 1 & 1.474 & 0.016 & 0.026 & 10.740 & 64.496
\end{tabular}

the dimensions and mosaicity of the specimen (Dietrich \& Als-Nielsen, 1965).

Studies on diffraction in mosaic crystals (e.g. Bacon \& Lowde, 1948; Zachariasen, 1969; Sears, 1997) have shown that the integrated intensity of the diffracted beam depends on the competitive processes that remove neutrons from the incident beam, i.e. Bragg diffraction $R(\lambda)$ on one side, and absorption and scattering $A(\lambda)+S(\lambda)$ on the other side. Hence, the integrated intensity measured for a given $h k l$ peak is expected to depend on the ratio between these two sources of beam attenuation. The comparison between these competing processes must be done over the wavelength range of the neutrons that contribute to the diffracted beam, e.g. $\left(\lambda_{h k l} \pm \varpi_{h k l} / 2\right)$. Hence, we have defined the parameter $\mathcal{M}_{h k l}$ given by

$$
\begin{aligned}
& \mathcal{M}_{h k l}=2 \frac{\int_{\lambda_{h k l}-\varpi_{h k l} / 2}^{\lambda_{h k l}+\omega_{\text {kinem }} / 2}(\lambda) \mathrm{d} \lambda}{\int_{\lambda_{h k l}-\Phi_{h k l} / 2}^{\lambda_{h k}+\Phi_{k h l}}[A(\lambda)+S(\lambda)] \mathrm{d} \lambda}
\end{aligned}
$$

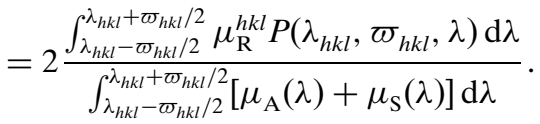

This $\mathcal{M}_{h k l}$ parameter can be calculated theoretically using the expressions presented in equations (7), (8), (9) and (13). Tables 2, 3 and 4 include the $\mathcal{M}_{h k l}$ parameters calculated for all peaks of the three specimens investigated, in all cases for the orientation $\phi=0^{\circ}$. Also included in the tables are the experimentally defined FWHM $\varpi_{h k l}$ for each reflection. Fig. 12 shows the dependence of the inverse of the extinction factor on the $\mathcal{M}_{h k l}$ parameter for the three specimens, which now displays a rather linear behaviour, captured by the solid straight lines that result from least-squares linear fits to the data. So, the $\mathcal{M}_{h k l}$ parameter provides a useful phenomenological description of the measured extinction factors, given by

$$
y_{h k l}=\mathcal{Y} / \mathcal{M}_{h k l} .
$$

Table 4

Refined and derived peak parameters for the pyrite crystal at $\phi=0^{\circ}$.

\begin{tabular}{rrrlllrr}
\hline$h$ & \multicolumn{1}{c}{$k$} & $l$ & $\lambda_{h k l}(\AA)$ & $y_{h k l}$ & $\varpi_{h k l}(\AA)$ & $\mathcal{M}_{h k l}$ & \multicolumn{1}{c}{$1 / y_{h k l}$} \\
\hline 2 & 0 & 1 & 4.428 & 0.133 & 0.032 & 0.144 & 7.519 \\
2 & 1 & 0 & 4.364 & 0.162 & 0.035 & 0.550 & 6.173 \\
2 & -1 & 0 & 4.290 & 0.158 & 0.037 & 0.528 & 6.329 \\
2 & 0 & -1 & 4.226 & 0.182 & 0.039 & 0.128 & 5.495 \\
1 & 1 & 1 & 3.835 & 0.021 & 0.003 & 37.380 & 47.619 \\
2 & 1 & 1 & 3.721 & 0.200 & 0.039 & 0.082 & 5.000 \\
1 & -1 & 1 & 3.712 & 0.017 & 0.003 & 62.268 & 58.824 \\
2 & -1 & 1 & 3.659 & 0.461 & 0.040 & 0.052 & 2.169 \\
2 & 1 & -1 & 3.552 & 0.172 & 0.042 & 0.078 & 5.814 \\
1 & 1 & -1 & 3.499 & 0.016 & 0.003 & 40.938 & 62.500 \\
2 & -1 & -1 & 3.491 & 0.180 & 0.044 & 0.048 & 5.556 \\
1 & -1 & -1 & 3.376 & 0.046 & 0.003 & 22.542 & 21.739 \\
3 & 0 & 1 & 3.296 & 0.191 & 0.016 & 0.122 & 5.236 \\
3 & 0 & -1 & 3.195 & 0.249 & 0.021 & 0.096 & 4.016 \\
3 & 1 & 1 & 3.013 & 0.062 & 0.021 & 5.822 & 16.129 \\
3 & -1 & 1 & 2.979 & 0.112 & 0.022 & 4.374 & 8.929 \\
3 & 1 & -1 & 2.921 & 0.197 & 0.024 & 2.550 & 5.076 \\
\hline & & & & & & &
\end{tabular}

Table 5

Linear coefficients $\mathcal{Y}$ in equation (25) obtained for the three specimens from the data shown in Fig. 12.

Also included in the table are the coefficients refined for the $\mathrm{Cu}$ monochromator measured at low temperatures.

\begin{tabular}{lc}
\hline & $\mathcal{Y}$ \\
\hline Cu monochromator & \\
Room temperature & 0.97 \\
$55 \mathrm{~K}$ & 0.99 \\
$70 \mathrm{~K}$ & 1.02 \\
$85 \mathrm{~K}$ & 1.03 \\
$135 \mathrm{~K}$ & 1.05 \\
$190 \mathrm{~K}$ & 1.05 \\
$225 \mathrm{~K}$ & 1.04 \\
Sikhote-Alin meteorite & 0.95 \\
Pyrite crystal & 1.05 \\
\hline
\end{tabular}

Table 5 lists the $\mathcal{Y}$ coefficients obtained for the three specimens from the data shown in Fig. 12 and those refined for the $\mathrm{Cu}$ monochromator measured at low temperatures. Rather surprisingly, we observe that the proportional constant $\mathcal{Y}$ is $\sim 1$ in all cases investigated, despite the differences in mosaicity among the three specimens and the different temperatures involved. This means that, as a first approximation, extinction factors could be calculated simply as $y_{h k l}=1 / \mathcal{M}_{h k l}$. A more detailed analysis of this finding is left for a future study, and the $\mathcal{M}_{h k l}$ parameter should be considered here only as a phenomenological correlation that can be included in the fullpattern model presented in this work.

Comparison of the $y_{h k l}^{-1}$ versus $\mathcal{M}_{h k l}$ plots of Fig. 12 reveals a better linear correlation for the $\mathrm{Cu}$ monochromator than for the natural specimens. This may be due to the more homogeneous microstructure of the monochromator, together with its very regular macroscopic shape and precise dimensions, which translate into a more predictable response of the passage of the neutrons through the material. Moreover, the shape of the specimen is quite close to the infinite slab usually considered in many theoretical analyses of this problem, so it 
is expected that relatively simple expressions could account for the observed extinction factors.

The proportional relation of equation (25) is in principle valid for the whole wavelength range; however, as is shown in Fig. 12, smaller $\mathcal{M}_{h k l}$ values exhibit some deviation from this linear behaviour. In all analysed cases, those values correspond to peaks found in the wavelength range where reflections are overlapping, and therefore the extinction factors are probably not very well defined. Moreover, for the optimal
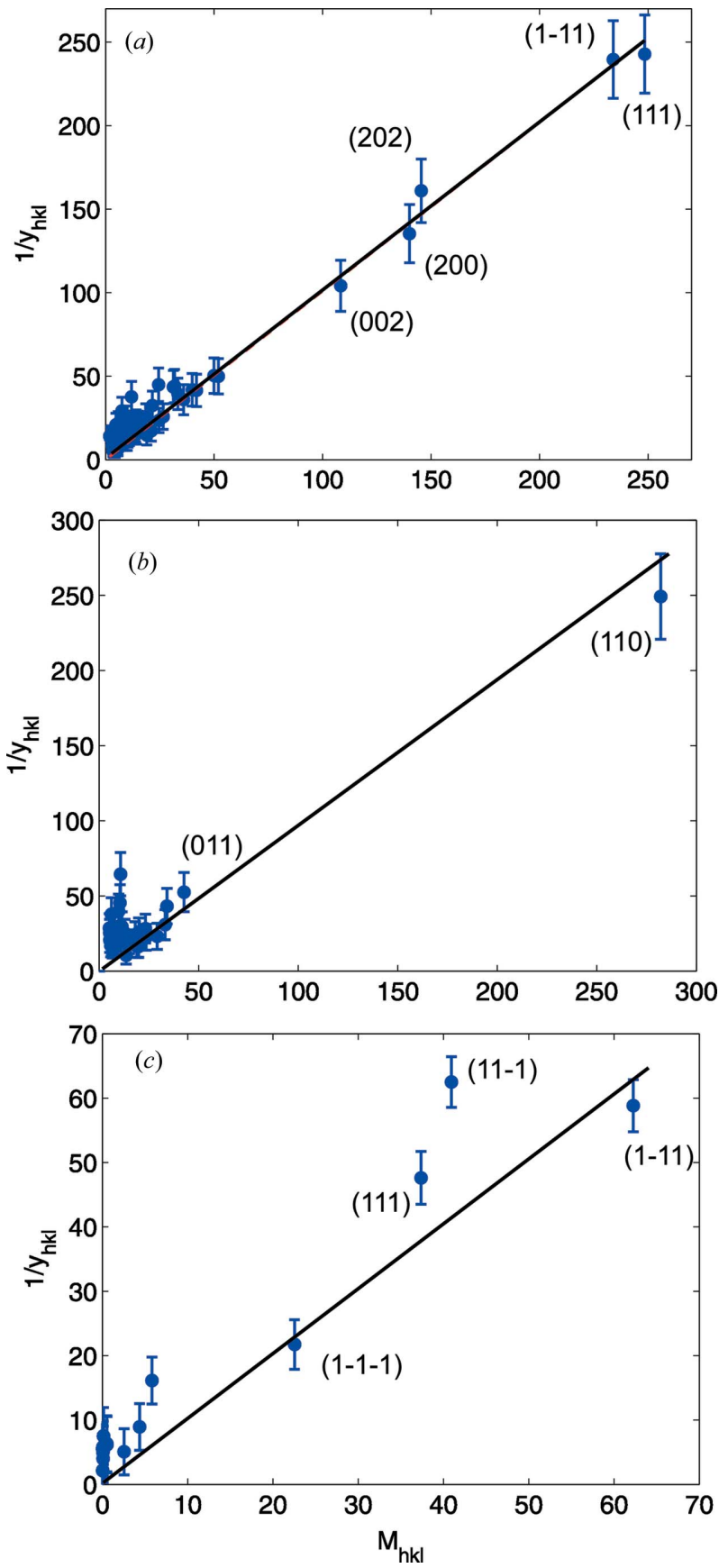

Figure 12

The dependence of the inverse of the extinction factor on the $\mathcal{M}_{h k l}$ parameter for the three specimens - the $\mathrm{Cu}$ monochromator specimen $(a)$, the NiFe meteorite $(b)$ and the natural pyrite sample $(c)$ - which displays a rather linear behaviour, captured by the solid straight lines that result from least-squares linear fits to the data.
Table 6

Linear coefficients $\mathcal{Y}$ calculated using the extinction factors as free fitting parameters or using equation (25) to constrain their values in the fullpattern refinement for the Sikhote-Alin meteorite.

\begin{tabular}{llllll}
\hline & \multicolumn{2}{l}{ Constrained $y_{h k l}$} & & \multicolumn{2}{l}{ Free $y_{h k l}$} \\
\cline { 2 - 3 } & $\mathcal{Y}$ & $G(\%)$ & & $\mathcal{Y}$ & $G(\%)$ \\
\hline $0^{\circ}$ & 0.98 & 5.1 & 0.97 & 4.1 \\
$2.5^{\circ}$ & 1.01 & 5.3 & 1.00 & 4.2 \\
$5^{\circ}$ & 1.05 & 5.5 & 0.99 & 4.1 \\
$10^{\circ}$ & 1.05 & 5.2 & & 0.99 & 4.0 \\
\hline
\end{tabular}

wavelength range found in $\S 5.1$ for the $\mathrm{Cu}$ monochromator at room temperature, $[1.5,4.5] \AA$, the minimum $\mathcal{M}_{h k l}$ value is 21 , and we see that in this case the linearity between $y_{h k l}$ and $\mathcal{M}_{h k l}$ holds for all Bragg peaks.

We have implemented the correlation between $y_{h k l}$ and $\mathcal{M}_{h k l}$ expressed in equation (25) in the analytical model used for the full-pattern least-squares refinement, which now becomes

$$
R(\lambda)=\sum_{h k l}\left(\mathcal{Y} / \mathcal{M}_{h k l}\right) \mu_{\mathrm{R}}^{h k l} P\left(\lambda_{h k l}, \varpi_{h k l}, \lambda\right)
$$

Use of this constraint between the integrated peak areas greatly reduces the number of fitting parameters, as only one parameter $(\mathcal{Y})$ is needed to describe the intensities of all the peaks appearing in $R(\lambda)$. For instance, for the refinement of the $\mathrm{Cu}$ monochromator shown in Fig. 4 the total number of fitting parameters is reduced from 85 to only 6 . Of course, this has some impact on the quality of the fitting and the associated $G$ parameter, which goes from 4.3 to $5.4 \%$. Fig. 13 shows the refinements performed in this way on the $R(\lambda)$ diffractograms measured for the samples at $\phi=0^{\circ}$ within the optimal wavelength range. The resulting $G$ value for the Sikhote-Alin meteorite is $13.1 \%$, which must be compared to the value of $7.7 \%$ obtained for the refinement shown in Fig. 7(b), performed with all peak intensities as free fitting parameters. Table 6 compares the values of $\mathcal{Y}$ and $G$ obtained using the constrained model with the values obtained when individual extinction factors are left as free fitting parameters for the Sikhote-Alin meteorite. In both situations the obtained $\mathcal{Y}$ values are $\sim 1$, and even when the constraint on extinction factors increases the $G$ value to $\sim 25 \%$, very good agreement is still found between the experimental $R(\lambda)$ patterns and the corresponding least-squares fits.

Note that inclusion of equation (25) in the full-pattern refinement does require the calculation of the $\mathcal{M}_{h k l}$ parameters defined in equation (24) prior to the least-squares fitting procedure, after indexing of the peaks. As seen before, the theoretical calculation of $R_{\text {kinem }}(\lambda)$ and $A(\lambda)+S(\lambda)$ involved in the definition of $\mathcal{M}_{h k l}$ requires knowledge of the Bragg angle for each particular reflection, as well as the incident beam divergence together with some estimation of the crystal mosaicity in order to evaluate the FWHM of the peak, $\varpi_{\mathrm{hkl}}$.

The phenomenological correlation found between peak areas and the $\mathcal{M}_{h k l}$ parameters opens the possibility of performing quantitative analyses of oligocrystals, i.e. speci- 
mens composed of a small number of crystals. In such cases, we should consider in the $R(\lambda)$ model one term for each crystal present in the specimen. Moreover, we have found that $\mathcal{Y}$ is $\sim 1$, so the peak intensities are just proportional to the thickness $(l)$ of the crystal along the neutron beam direction. Then, for a specimen composed of $N_{\mathrm{c}}$ crystals we have

$$
R(\lambda)=\sum_{j=1}^{N_{\mathrm{c}}} l_{j}\left[\sum_{h k l}\left(\mu_{\mathrm{R}}^{h k l} / \mathcal{M}_{h k l}\right) P\left(\lambda_{h k l}, \varpi_{h k l}, \lambda\right)\right],
$$

where $j$ runs over the crystals. By performing a full-pattern least-squares refinement using the crystal thicknesses $l_{j}$ as
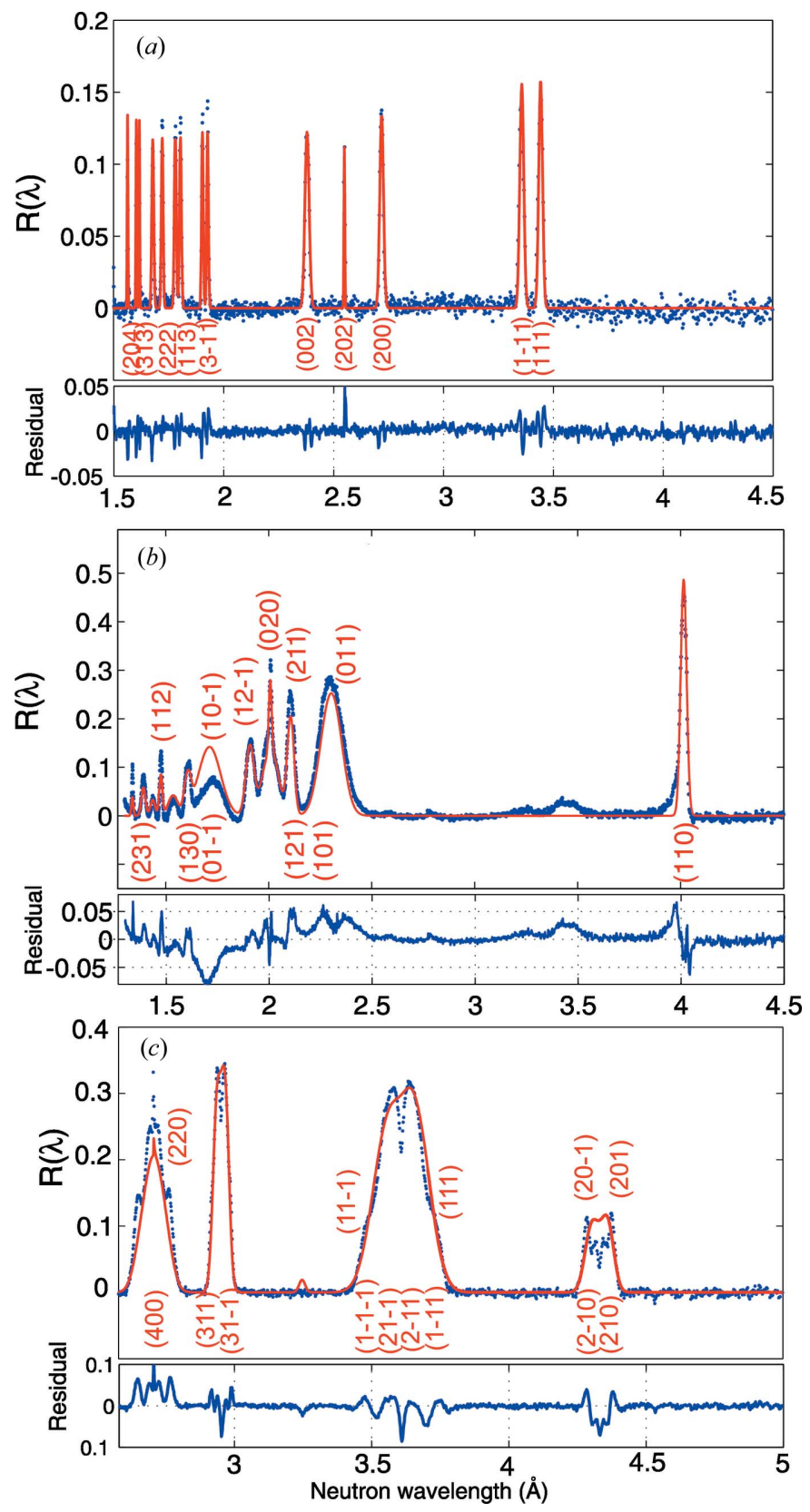

Figure 13

Full-profile refinement employing the constraint between the integrated peak areas [equation 26)] on the $R(\lambda)$ diffractogram measured for the $\mathrm{Cu}$ monochromator specimen $(a)$, the Sikhote-Alin meteorite $(b)$ and the natural pyrite sample $(c)$, at $\phi=0^{\circ}$. fitting variables, it is possible to determine the volume fraction of the different crystals composing the specimen. Hence, a quantitative texture analysis is in principle possible for specimens composed of crystals of only one phase, whilst a quantitative phase analysis is available for specimen formed by several phases, such as turbine blades made of nickel superalloys and, possibly, the Sikhote-Alin meteorite investigated here. Further studies are required for a full assessment of the possibilities offered by the proposed method for the study of oligocrystals.

\section{Conclusions}

We measured the room-temperature wavelength-dependent neutron transmission of two naturally occurring mosaic crystal specimens having thicknesses of $\sim 10 \mathrm{~mm}$, i.e. an iron-nickel meteorite from the Sikhote-Alin fall and a pyrite crystal. We also measured the transmission of a man-made $\mathrm{Cu}$ crystal, used as a monochromator in neutron instruments, at temperatures between $55 \mathrm{~K}$ and room temperature.

In all cases, the transmission presented a number of dips at specific neutron wavelengths, due to Bragg reflection on the crystal planes, together with an overall reduction in intensity due to absorption and scattering processes. The position, intensity and width of the dips were strongly dependent on the orientation of the specimen in the neutron beam.

All measured transmission spectra were properly described by a full-pattern model that includes the most important variables of the experiment, e.g. the crystal structure, dimensions, mosaicity and orientation of the specimen, and the divergence and wavelength resolution of the instrument. An efficient least-squares refinement of the experimental data using the proposed model provided precise values of lattice parameters, mosaicities and crystal orientation.

The effect of extinction was evident from the measured intensity of the Bragg-reflected beams, and it was quantified by means of an extinction factor associated with each reflection. Overall, these extinction factors were experimentally found to correspond to the ratio between the kinematical attenuation coefficient due to reflection on the crystal planes and the combined attenuation coefficient including absorption and scattering processes. By properly accounting for secondary extinction on the transmitted beam, it should become possible to perform quantitative analysis of crystallographic phases and texture on specimens formed by a small number of crystals.

The full-pattern analysis proposed here should find direct application in energy-resolved neutron imaging instruments, hence expanding the range of investigations performed by that novel technique.

\section{APPENDIX $A$}

The calculation of the scattering contribution $\mu_{\mathrm{S}}(\lambda)$ to the attenuation coefficient is a small variation from the recommendations given by Granada (1984). For a monoatomic crystal we have 


$$
\mu_{\mathrm{S}}(\lambda)=N \sigma_{\mathrm{S}}\left(\lambda, \sigma_{\mathrm{coh}}, \sigma_{\text {inc }}, \mathcal{A}, \Theta_{\mathrm{D}}, T\right)
$$

with $N$ the number of atoms per unit volume and $\sigma_{\mathrm{s}}$ the associated microscopic cross section, which includes a combination of elastic incoherent scattering processes and inelastic scattering.

$$
\sigma_{\mathrm{S}}=\sigma_{\text {inc }}^{\mathrm{el}}+\sigma_{\text {coh }}^{\text {inel }}+\sigma_{\text {inc }}^{\text {inel }}=\sigma_{\text {inc }} S_{\text {inc }}^{\text {el }}+\sigma_{\text {coh }} S_{\text {coh }}^{\text {inel }}+\sigma_{\text {inc }} S_{\text {inc }}^{\text {inel }},
$$

where $\sigma_{\text {inc }}$ and $\sigma_{\text {coh }}$ are the incoherent and coherent nuclear constants, respectively, while the functions $S$ contain the details of the structure and dynamics of the sample system (Squires, 1978). Assuming that the crystal vibrations are described by a Debye frequency spectrum of characteristic temperature $\Theta_{\mathrm{D}}$, the elastic incoherent part of the cross section is given by

$$
S_{\text {inc }}^{\mathrm{el}}\left(\lambda, \mathcal{A}, \Theta_{\mathrm{D}}, T\right)=\frac{\lambda^{2}}{16 \pi^{2}\left\langle u^{2}\right\rangle}\left[1-\exp \left(-\frac{16 \pi^{2}}{\lambda^{2}}\left\langle u^{2}\right\rangle\right)\right],
$$

where $\left\langle u^{2}\right\rangle$ is the root-mean-square displacement of an atom, which is the same parameter as determinates the DebyeWaller factor. This parameter is a function of the atomic mass $\mathcal{A}$, the Debye temperature $\Theta_{\mathrm{D}}$ and the specimen temperature $T$, and can be calculated for elemental crystals using Sears \& Shelley (1991) expressions.

The calculation of the inelastic scattering components is complicated. Hence, the so-called 'incoherent approximation' is usually adopted. This consists of calculating the incoherent inelastic contribution and postulating that the coherent elastic contribution has the same dependence on $\left(\lambda, \mathcal{A}, \Theta_{\mathrm{D}}, T\right)$, but its scattering power is given by the coherent nuclear constant $\sigma_{\text {coh }}$ rather than by $\sigma_{\text {inc }}, \quad$ e.g. $\quad S_{\text {coh }}^{\text {inel }}\left(\lambda, \mathcal{A}, \Theta_{\mathrm{D}}, T\right)=$ $S_{\text {inc }}^{\text {inel }}\left(\lambda, \mathcal{A}, \Theta_{\mathrm{D}}, T\right)$. So, the total inelastic scattering becomes

$$
\sigma_{\text {tot }}^{\text {inel }}=\left(\sigma_{\text {coh }}+\sigma_{\text {inc }}\right) S_{\text {inc }}^{\text {inel }}\left(\lambda, \mathcal{A}, \Theta_{\mathrm{D}}, T\right)
$$

The incoherent inelastic scattering function $S_{\text {inc }}^{\text {inel }}$ is calculated as

$$
S_{\text {inc }}^{\text {inel }}\left(\lambda, \mathcal{A}, \Theta_{\mathrm{D}}, T\right)=S_{\text {inc }}^{\mathrm{tot}}\left(\lambda, \mathcal{A}, \Theta_{\mathrm{D}}, T\right)-S_{\text {inc }}^{\mathrm{el}}\left(\lambda, \mathcal{A}, \Theta_{\mathrm{D}}, T\right)
$$

with $S_{\text {inc }}^{\text {tot }}$ the total incoherent scattering function calculated by means of a series in the inverse of the atom mass (Placzek, 1952).

$$
S_{\mathrm{inc}}^{\mathrm{tot}}\left(\lambda, \mathcal{A}, \Theta_{\mathrm{D}}, T\right)=\sum_{n=0}^{\infty}(m / \mathcal{A})^{n} A_{n}(\lambda, \Theta)
$$

where $m$ is the neutron mass, $\Theta=T / \Theta_{\mathrm{D}}$,

$$
\left\{\begin{array}{l}
A_{n}(\lambda, \Theta)=\frac{1}{4 x^{2}} \frac{3^{n}}{n+1} \sum_{l=0}^{n} a_{l}^{(n)} \\
A_{0}(\lambda, \Theta)=1
\end{array}\right.
$$

and

$$
\begin{aligned}
& a_{l}^{(n)}=\frac{\left(-\frac{2 \mathcal{A} k_{\mathrm{B}} \Theta_{\mathrm{D}}}{3 \hbar^{2}}\left\langle u^{2}\right\rangle\right)^{n-l}}{l !(n-l) !} \\
& \times \prod_{j=1}^{l} \int_{-1}^{1} \mathrm{~d} \mathcal{E}_{j} \frac{\mathcal{E}_{j}}{\exp \left(\mathcal{E}_{j} / \Theta\right)-1}\left[\left(x+x^{\prime}\right)^{2 n+2}-\left(x-x^{\prime}\right)^{2 n+2}\right],
\end{aligned}
$$

with $k_{\mathrm{B}}$ the Boltzmann constant and $\hbar$ Planck's constant over $2 \pi$. The definition of the dimensionless parameters $x$ and $x^{\prime}$, is as follows:

$$
\begin{gathered}
x^{2}=\frac{2 \pi^{2} \hbar^{2}}{m k_{\mathrm{B}} \Theta_{\mathrm{D}} \lambda^{2}}, \\
x^{\prime}= \begin{cases}\left(x^{2}+\sum_{j=1}^{l} \mathcal{E}_{j}\right)^{1 / 2} & \text { if } \sum_{j=1}^{l} \mathcal{E}_{j}>-x^{2}, \\
0 & \text { otherwise. }\end{cases}
\end{gathered}
$$

Summarizing, the scattering contribution to the attenuation coefficient is calculated as

$$
\begin{aligned}
\mu_{\mathrm{S}}(\lambda)= & N\left\{\sigma_{\text {inc }} \mathrm{S}_{\text {inc }}^{\mathrm{el}}\left(\lambda, \mathcal{A}, \Theta_{\mathrm{D}}, T\right)+\left(\sigma_{\text {coh }}+\sigma_{\text {inc }}\right)\right. \\
& \left.\times\left[S_{\text {inc }}^{\mathrm{tot}}\left(\lambda, \mathcal{A}, \Theta_{\mathrm{D}}, T\right)-S_{\text {inc }}^{\mathrm{el}}\left(\lambda, \mathcal{A}, \Theta_{\mathrm{D}}, T\right)\right]\right\} .
\end{aligned}
$$

\section{Acknowledgements}

The authors would like to thank Lucia Alianeli for help and useful discussion on the $\mathrm{Cu}$ monochomator work. This research was partially funded by CONICET under PIP-5422011.

\section{References}

Antonijevic, M. M., Mihajlovic, R. P. \& Vukanovic, B. V. (2002). Sensors, 2, 153-163.

Bacon, G. E. \& Lowde, R. D. (1948). Acta Cryst. 1, 303-314.

Bastie, P., Hamelin, B. \& Courtois, P. (2000). J. Phys. IV 10, Pr10-21Pr10-26.

Birkholz, M. (1992). J. Phys. Condens. Matter, 4, 6227-6240.

Bragg, W. L. (1914). Proc. R. Soc. Lond. Ser. A, 89, 476-478.

Buchwald, V. F. (1976). Handbook of Iron Meteorites. Their History, Distribution, Composition and Structure. Berkeley: University of California Press.

Bunge, H. J. (1982). Texture Analysis in Materials Science: Mathematical Methods. London: Butterworths.

Courtois, P., Andersen, K. H. \& Bastie, P. (2006). Exp. Astron. 20, 195-200.

Darwin, C. G. (1922). Philos. Mag. Ser. 6, 43, 800-829.

Dietrich, O. W. \& Als-Nielsen, J. (1965). Acta Cryst. 18, 184-188.

Echarri, A. L. \& Sánchez, C. (1974). Solid State Commun. 15, 827831.

Ferrer, I. J. \& Sánchez, C. (1992). Solid State Commun. 81, 371374.

Gale, W. F. \& Totemeier, T. C. (2004). Editors. Smithells Metals Reference Book. Amsterdam: Elsevier.

Gallant, R. (1996). Meteor. Mag. 2, 8-11.

Granada, J. R. (1984). Z. Naturforsch. 39, 1160-1167. 
Hamilton, W. C. (1957). Acta Cryst. 10, 629-634.

Harjo, S., Aizawa, K., Ito, T., Arima, H., Abe, J., Moriai, A., Sakasai, K., Nakamura, T., Nakatani, T., Iwahashi, T. \& Kamiyama, T. (2010). Mater. Sci. Forum, 652, 99-104.

He, Y., Godet, S., Jacques, P. J. \& Jonas, J. J. (2006). Acta Mater. 54, 1323-1334.

Hielscher, R. \& Schaeben, H. (2008). J. Appl. Cryst. 41, 10241037.

Kiyanagi, Y., Sato, H., Kamiyama, T. \& Shinohara, T. (2012). J. Phys. Conf. Ser. 340, 012010.

Kockelmann, W., Frei, G., Lehmann, E. H., Vontobel, P. \& Santisteban, J. R. (2007). Nucl. Instrum. Methods Phys. Res.Sect. $A, \mathbf{5 7 8}, 421-434$.

Kockelmann, W., Zhang, S. Y., Kelleher, J. F., Nightingale, J. B., Burca, G. \& James, J. A. (2013). Phys. Procedia, 43, 100-110.

Kropff, F., Granada, J. R. \& Mayer, R. E. (1982). Nucl. Instrum. Methods Phys. Res. 198, 515-521.

Makowska, M. G., Strobl, M., Lauridsen, E. M., Frandsen, H. L., Tremsin, A. S., Kardjilov, N., Manke, I., Kelleher, J. F. \& Theil Kuhn, L. (2015). J. Appl. Cryst. 48, 401-408.

Malamud, F., Santisteban, J. R., Vicente Alvarez, M. A., Bolmaro, R., Kelleher, J., Kabra, S. \& Kockelmann, W. (2014). J. Appl. Cryst. 47, 1337-1354.

McKeehan, L. W. (1923). Phys. Rev. 21, 402-407.

Murowchick, J. B. \& Barnes, H. L. (1987). Am. Mineral. 72, 12411250.

Murphy, R. \& Strongin, D. R. (2009). Surf. Sci. Rep. 64, 1-45.

Owen, E. A. \& Burns, B. D. (1939). London, Edinb. Dubl. Philos. Mag. J. Sci. 28, 497-519.

Owen, E. A., Yates, E. L. \& Sully, A. H. (1937). Proc. Phys. Soc. 49, 315-322.

Placzek, G. (1952). Phys. Rev. 86, 377-388.

Reisener, R. J. \& Goldstein, J. I. (2003). Meteorit. Planet. Sci. 38, 1669-1678.

Rietveld, H. M. (1969). J. Appl. Cryst. 2, 65-71.

Santisteban, J. R. (2005). J. Appl. Cryst. 38, 934-944.

Santisteban, J. R., Daymond, M. R., James, J. A. \& Edwards, L. (2006). J. Appl. Cryst. 39, 812-825.
Santisteban, J., Edwards, L., Fitzpatrick, M., Steuwer, A., Withers, P., Daymond, M., Johnson, M., Rhodes, N. \& Schooneveld, E. (2002). Nucl. Instrum. Methods Phys. Res. Sect. A, 481, 765-768.

Santisteban, J. R., Vicente-Alvarez, M. A., Vizcaino, P., Banchik, A. D., Vogel, S. C., Tremsin, A. S., Vallerga, J. V., McPhate, J. B., Lehmann, E. \& Kockelmann, W. (2012). J. Nucl. Mater. 425, 218-227.

Sato, K. (1985). Prog. Cryst. Growth Charact. 11, 109-154.

Schieck, R., Hartmann, A., Fiechter, S., Könenkamp, R. \& Wetzel, H. (1990). J. Mater. Res. 5, 1567-1572.

Schlegel, A. \& Wachter, P. (1976). J. Phys. C Solid State Phys. 9, 33633369.

Sears, V. F. (1997). Acta Cryst. A53, 35-45.

Sears, V. F. \& Shelley, S. A. (1991). Acta Cryst. A47, 441-446.

Simmons, R. O. \& Balluffi, R. W. (1957). Phys. Rev. 108, 278-280.

Squires, G. L. (1978). Introduction to the Theory of Thermal Neutron Scattering. Mineola: Dover Publications.

Steuwer, A., Santisteban, J., Withers, P. \& Edwards, L. (2004). Phys. B Condens. Matter, 350, 159-161.

Steuwer, A., Santisteban, J. R., Withers, P. J., Edwards, L. \& Fitzpatrick, M. E. (2003). J. Appl. Cryst. 36, 1159-1168.

Strobl, M., Grünzweig, C., Hilger, A., Manke, I., Kardjilov, N., David, C. \& Pfeiffer, F. (2008). Phys. Rev. Lett. 101, 123902.

Strobl, M., Woracek, R., Kardjilov, N., Hilger, A., Wimpory, R., Tremsin, A., Wilpert, T., Schulz, C., Manke, I. \& Penumadu, D. (2012). Nucl. Instrum. Methods Phys. Res. A, 680, 27-34.

Treimer, W., Strobl, M., Kardjilov, N., Hilger, A. \& Manke, I. (2006). Appl. Phys. Lett. 89, 203504.

Tremsin, A. S., McPhate, J. B., Steuwer, A., Kockelmann, W. M., Paradowska, A., Kelleher, J. F., Vallerga, J. V., Siegmund, O. H. W. \& Feller, W. B. (2012). Strain, 48, 296-305.

Vogel, S. (2000). PhD thesis, Christian-Albrechts Universität Kiel, Germany.

Woracek, R., Penumadu, D., Kardjilov, N., Hilger, A., Boin, M., Banhart, J. \& Manke, I. (2014). Adv. Mater. 26, 4069-4073.

Zachariasen, W. H. (1945). Theory of X-ray Diffraction in Crystals. New York: John Wiley and Sons.

Zachariasen, W. H. (1967). Acta Cryst. 23, 558-564.

Zachariasen, W. H. (1969). Acta Cryst. A25, 102. 